\title{
Infections in Liver Transplantation
}

\author{
B. Sharmila Mohanraj, Amol S. Rangnekar, \\ and Joseph G. Timpone Jr.
}

\section{Indications for Liver Transplantation}

Orthotopic liver transplantation (LT) is most commonly offered to patients with end-stage liver disease. However, this lifesaving therapy can also be used to successfully treat patients with acute liver failure, primary and some metastatic liver tumors, and selected metabolic conditions (Table 3.1).

Decompensated cirrhosis is defined by the presence of specific complications including jaundice, hepatic encephalopathy, ascites, spontaneous bacterial peritonitis, hepatorenal syndrome, or variceal hemorrhage. Once a patient develops complications of portal hypertension, 5-year survival is $<50 \%$. Additionally, patients with cirrhosis may develop life-threatening pulmonary complications such as hepatopulmonary syndrome (HPS) or portopulmonary hypertension (PPH).

The Child-Turcotte-Pugh (CTP) score helps to stratify severity of illness according to a combination of five physiologic and laboratory variables: ascites, hepatic encephalopathy, bilirubin, albumin, and prothrombin time. Patients in CTP class B or C have less than a $60 \%$ 2-year survival and should be considered for OLT. The Model for End-Stage Liver Disease (MELD) score is a more simplified and objective method designed to characterize the degree of illness of patients with end-stage liver disease. The MELD score incorporates serum bilirubin, prothrombin time, and creatinine values [1]. Based on its ability to predict survival, the MELD score has been used to prioritize patients on the OLT wait list. It is generally accepted that patients with a MELD score greater than 10 should be referred for liver

B. S. Mohanraj · J. G. Timpone Jr. ( $\square)$

MedStar Georgetown University Hospital, Division of Infectious

Diseases and Travel Medicine, Washington, DC, USA

e-mail: bernardine.r.s.mohanraj@gunet.georgetown.edu;

timponej@gunet.georgetown.edu

\section{A. S. Rangnekar}

MedStar Georgetown University Hospital, MedStar Georgetown

Transplant Institute, Washington, DC, USA

e-mail: amol.s.rangnekar@gunet.georgetown.edu
Table 3.1 Indications for liver transplantation

Decompensated hepatic cirrhosis

Biliary cirrhosis (primary biliary cholangitis, primary sclerosing cholangitis, biliary atresia, Alagille syndrome, cystic fibrosis, progressive familial intrahepatic cholestasis)

Hepatocellular carcinoma

Hepatoblastoma

Hemangioendothelioma

Metastatic neuroendocrine tumors

Glycogen storage disease

Neonatal hemochromatosis

Amyloidosis

Hyperoxaluria

Urea cycle defects

Disorders of branch chain amino acids

Acute liver failure

Budd-Chiari syndrome

Polycystic liver disease

transplant evaluation, and those with a MELD score of 15 or higher are most likely to derive benefit from OLT [2]. Despite its simplicity, the MELD score does disadvantage a subset of patients who have severely decompensated liver disease but minimally abnormal laboratory results. Recently, serum sodium has been incorporated into the MELD score, i.e., MELD-Na. Hyponatremia is an independent predictor of mortality in patients with decompensated cirrhosis, and diminished serum sodium levels may be a surrogate marker of advanced portal hypertension [3, 4].

Acute liver failure (ALF) is a rare but life-threatening condition, which is manifested by evidence of hepatic injury, coagulopathy, encephalopathy, and absence of underlying cirrhosis in most patients. In Western countries, nearly half of all cases are attributed to acetaminophen overdose. Other less common etiologies include drug injury, viral hepatitis, autoimmune hepatitis, and fulminant Wilson disease. Mortality exceeds $30 \%$ with death often occurring within 1 week of presentation. Although a majority of patients with ALF due to acetaminophen toxicity may recover spontaneously, those with ALF due to other etiologies often require OLT to survive $[5,6]$. 
Certain patients with liver-based metabolic conditions and systemic complications may also benefit from OLT. Examples of these conditions include familial amyloidosis, glycogen storage disease, and primary hyperoxaluria. Although underlying liver synthetic function is preserved, hepatic allograft transplant allows for correction of a specific metabolic deficit in these patients.

As the incidence of hepatocellular carcinoma (HCC) continues to rise, patients with this disease now represent a substantial proportion of liver transplant recipients. Patients with cirrhosis and portal hypertension are unlikely to tolerate hepatic resection of their HCC and are offered OLT in order to improve their recurrence-free survival. Typically, patients with tumor burden within the Milan criteria are considered good candidates for OLT and are typically awarded MELD exception points [7]. More liberal tumor burden criteria have been proposed for transplant, although larger tumor size and number seem to correlate with a higher risk of recurrence [8].

Most patients with cholangiocarcinoma have traditionally not been candidates for liver transplants due to high post-transplant recurrence rates and poor survival. However, recent data suggest that carefully selected patients with isolated unresectable hilar tumors who are treated with neoadjuvant chemotherapy and radiation therapy may have acceptable survival rates after transplant [9]. In general, patients with known intrahepatic cholangiocarcinoma may be candidates for resection but are not typically considered for OLT.

In the USA, patients are prioritized for liver transplantation on the basis of the MELD score. In selected cases, patients with specific complications of liver disease may be eligible for MELD exception scores. MELD exception scores may be standardized, as is the case presently in patients with HCC within Milan criteria, certain pulmonary complications of cirrhosis, and selected metabolic disorders. In specific cases, some patients may be granted MELD exception on an individual basis by the regional review board after taking into account extenuating circumstances.

\section{Contraindications to Liver Transplant}

Life-threatening conditions such as severe cardiopulmonary disease or sepsis are generally major contraindications to OLT. Although patients with human immunodeficiency virus (HIV) infection can successfully undergo transplant, those with AIDS are thought to be poor candidates based on their poor health and the risk of additional post-transplant immune suppression. Patients actively using drugs or alcohol are often excluded as well. Many transplant centers have traditionally required abstinence from alcohol for a minimum of 6 months prior to listing, although this requirement has been evolving recently after a significant post-transplant survival benefit was demonstrated in selected patients with severe alcoholic hepatitis [10]. Patients with extrahepatic malignancy, metastatic HCC, or intrahepatic cholangiocarcinoma should not undergo transplant. Patients may also be denied liver transplant on the basis of certain psychosocial factors such as persistent noncompliance with medical care or lack of adequate social support. Relative contraindications to liver transplantation include advanced age, severe obesity, prior abdominal surgeries, and significant mesenteric vascular thromboses.

Pre-transplant infectious disease work-up includes serologic testing to diagnose infectious causes of acute or chronic liver disease such as hepatitis A virus (HAV), hepatitis $\mathrm{B}$ virus (HBV), and hepatitis $\mathrm{C}$ virus (HCV) and to identify latent infections, which may reactivate in the setting of post-transplant immunosuppression. These include cytomegalovirus (CMV), Epstein-Barr virus (EBV), varicella zoster virus (VZV), rapid plasma regain test for syphilis, and interferon gamma release assay or tuberculin skin test for latent tuberculosis. All patients are screened for HIV. Selected high-risk individuals may undergo testing for coccidioidomycosis, Trypanosoma cruzi, or Strongyloides stercoralis, depending upon geographic location, travel history, and/or history of exposure to various endemic infections. Pre-transplant dental evaluation is typically mandatory in order to identify and manage potential oral sources of infection after undergoing transplantation. Dental extractions, if warranted, should occur prior to liver transplantation. Immunizations for $\mathrm{HAV}, \mathrm{HBV}$, pneumococcus, influenza, diphtheria, tetanus, and pertussis should be administered to appropriate transplant candidates at the time of the pre-transplant evaluation. As there is a contraindication to live vaccines after transplantation, immunization status for measles, mumps, and rubella (MMR) and varicella should also be obtained. The American Association for the Study of Liver Diseases (AASLD) has published online guidelines in 2013 for the evaluation of potential liver transplant recipients which can be found at https://www.aasld. org/publications/practice-guidelines-0.

Based on reasonable short- and long-term outcomes, patients with HIV infection can successfully undergo OLT. Typically, these patients should have well-controlled disease on antiretroviral therapy, with undetectable HIV RNA and absence of active AIDS-associated opportunistic infections or malignancies. CD4+ counts $>100$ may be a reasonable cutoff in patients with leukopenia due to portal hypertension and splenic sequestration. Other contraindications to liver transplantation may include a history of AIDS-defining opportunistic infection that requires chronic secondary prophylaxis or has limited treatment options such as progressive multifocal leukoencephalopathy (PML), disseminated Mycobacterium avium complex, chronic cryptosporidiosis, 
EBV and human herpesvirus-8-related lymphoproliferative disorders such as non-Hodgkin's lymphoma and visceral Kaposi's sarcoma (KS), or significant cervical or anal neoplastic disease due to human papillomavirus. Some centers have performed transplants in patients with cutaneous KS. Although recurrence of the malignancy can occur after undergoing transplant surgery, these patients may be managed with mechanistic target of rapamycin (mTOR) inhibitor-based immunosuppressive regimens after OLT based on the antineoplastic effects of these agents.

Several factors may increase the risk for graft loss in $\mathrm{HIV}+$ patients undergoing liver transplants including older donor age, HCV-positive donors, low recipient BMI, and simultaneous liver and kidney transplantation [11]. Of note, these outcomes and predictors of graft loss were identified in older studies of HIV- and HCV-coinfected patients undergoing transplant. Additional studies are needed to determine outcomes in an era of improved HCV therapy and specifically in non-coinfected patients requiring transplant.

\section{Types of Liver Transplantation}

The majority of liver transplants in the Western world utilize whole organ allografts from deceased donors. However, alternative options such as split deceased donor grafts or partial living donor grafts are used occasionally. In the USA, living donor liver transplantation (LDLT) currently accounts for less than $5 \%$ of all liver transplants, although in other parts of the world, LDLT is utilized more frequently.

Most deceased donors are considered on the basis of donation after brain death (DBD). Occasionally donation after circulatory death (DCD) can be considered. DCD donors are characterized by the absence of systemic circulation along with irreversible apnea and unresponsiveness. The main risk associated with DCD is organ ischemia and the potential for biliary epithelial injury. Initial results of the experience with DCD OLT were marked by increased rates of ischemic cholangiopathy, a condition which can lead to recurrent cholangitis, graft loss requiring retransplantation, or death $[12,13]$. However, some centers have reported similar patient and graft survival with DCD and DBD donors, particularly with the use of donors less than 40 years old, and minimization of cold and warm ischemia times [14, 15].

LDLT offers the advantage of allowing for expedited access to transplantation at a time before the recipient develops advanced liver failure while simultaneously increasing the donor pool. However, these benefits must be balanced against the potential risks of morbidity and death for the donor.

In order to be considered for LDLT in the USA, patients must already be listed for deceased donor liver transplantation (DDLT). Selected recipients with low MELD score and significant hepatic decompensation, HCC, or other complications of advanced liver disease such as primary sclerosing cholangitis with recurrent episodes of ascending cholangitis or polycystic liver disease are typically good candidates for LDLT. Conversely, patients with higher MELD scores and severe hepatic decompensation usually have limited survival and a shorter anticipated waiting time for transplant. As compared to patient undergoing DDLT, LDLT recipients may have a significantly higher rate of complications and need for retransplantation, although adjusted long-term survival appears to be similar $[16,17]$. As such, careful recipient selection is critical. Contraindications to LDLT may include significant mesenteric vascular thrombosis, severe portal hypertension, need for simultaneous renal transplantation, or retransplantation. An additional consideration is matching donor and recipient body size. Specifically, a graft weightto-body weight ratio of $0.8 \%$ is recommended to avoid the development of small-for-size syndrome, a condition marked by the development of hyperbilirubinemia, intractable ascites, coagulopathy, renal failure, and extended duration of hepatic encephalopathy. Biliary complications tend to occur with higher frequency in LDLT recipients, although rates are lower in centers with higher volume living donor, partial hepatic graft transplantation [18].

Deceased donor livers may also be split to maximize yield for recipients. As with LDLT, adequate graft weight for the recipient must be determined prior to transplant. Most commonly deceased donor livers are split so as to offer the left lateral segment to a pediatric recipient and the remaining allograft to an adult recipient.

\section{Donor Selection}

In the USA, the majority of liver donation occurs after brain death. The criteria to determine initial DBD donor eligibility include complete apnea, brainstem areflexia, cerebral unresponsiveness, and evidence of irreversible and permanent loss of central nervous system function [19, 20]. The diagnosis can often be determined based on clinical examination and noninvasive testing such as electroencephalography, evoked cerebral potentials, and transcranial Doppler ultrasonography [21, 22].

Absolute contraindications to organ donation include most extrahepatic malignancies and transmissible infections associated with high risk of mortality. Specifically, donors with active extracranial or hematologic malignancies are not considered suitable for organ donation. However, patients with primary brain tumors without extracranial metastases may be considered as donors for transplant. Donors with fungemia, mycobacterial disease, disseminated resistant bacterial infections, and prion disease are typically excluded from donation. 
Once a donor is considered suitable based on these preliminary criteria, additional information is required. Initial donor-recipient matching is determined based primarily on blood type and graft size, although multiple other factors are evaluated prior to transplantation. An ideal donor would meet the following criteria: age less than 50 years, hemodynamic stability, absence of significant chronic disease, systemic infection, malignancy, or abdominal trauma.

However, since only a limited number of donors meet these parameters, transplant providers have liberalized exclusion criteria in an effort to increase the donor pool. Extended criteria donor grafts refer to those from donors with older age, higher degree of hepatic steatosis, history of malignancy, active viral or bacterial infection, or history of trauma. Traditionally, older donor age was considered a risk factor for graft failure, primarily based on increased risk for chronic vascular disease, significant comorbidities, and potentially more hepatic steatosis in elderly donors. However, more recent experiences suggest that donor age alone may not be a predictor of graft outcome. In fact, older donors even greater than 80 years old may be used with reasonable success if other donor risk factors such as hepatic steatosis and cold ischemia time can be minimized [23-25]. The use of older donors in recipients with HCV infection has traditionally correlated with increased graft loss. This finding may become less relevant in the modern era of improved $\mathrm{HCV}$ direct antiviral therapy, as HCV infection can be successfully eradicated in the majority of HCV-seropositive recipients either before or soon after liver transplantation.

Hepatic steatosis can be quantified by biopsy of the donor liver. Grafts with mild macrovesicular steatosis $(<30 \%)$ are routinely used with good graft outcomes. In contrast, severe macrovesicular steatosis $(>60 \%)$ within the donor graft increases the risk of primary nonfunction and is usually a contraindication for use of the organ [26, 27]. The donor risk index (DRI) predicts liver graft failure based on specific donor characteristics and transplant factors including age, race, height, cause of death, partial or split organ, DCD status, cold ischemia time, and sharing outside of a local donor service area [28].

All organ donors undergo nucleic acid testing (NAT) to rule out specific transmissible viral infections such as HIV, $\mathrm{HBV}$, or HCV. In general there is a finite, albeit low, risk of viral transmission from the average donor. A study of organ procurement organizations in the USA has reported $\mathrm{HIV}$ and $\mathrm{HCV}$ prevalence rates of $0.1 \%$ and $3.5 \%$, respectively, in donors with normal risk. Among high-risk donors, the prevalence of HIV and HCV was higher $(0.5 \%$ and $18 \%$, respectively). A model based on these known prevalence numbers estimates the incidence of undetected viremia as being 1 in 60,000 for HIV and 1 in 5000 for HCV in normalrisk donors. Among high-risk donors, the incidence may rise to 1 in 12,000 for HIV and 1 in 1000 for HCV [29].
The US Public Health Service (PHS) has developed specific criteria to classify donors as being at increased risk for recent HIV, HBV, or HCV infection [30]. These criteria include factors such as high-risk sexual behaviors and prior injection drug use. The specific PHS criteria for high-risk donors established in 2013 include:

1. High-risk sexual behavior within the preceding 12 months (people who have had sex with a person known or suspected to have HIV, HBV, or HCV infections, men who had sex with men [MSM], women who have had sex with an MSM, people who have had sex in exchange for money or drugs, people who have had sex with a person who had sex in exchange for money or drugs, people who have had sex with a person that has injected drugs by intravenous, intramuscular, or subcutaneous route for nonmedical reasons)

2. High-risk children (a child who is $\leq 18$ months of age and born to a mother known to be infected with or at increased risk for $\mathrm{HIV}, \mathrm{HBV}$, or $\mathrm{HCV}$ infections, a child who has been breastfed within the preceding 12 months and a mother known to be infected with, or at increased risk for, HIV infection)

3. People who have injected drugs by intravenous, intramuscular, or subcutaneous route for nonmedical reasons in the preceding 12 months

4. People who have been in lockup, jail, prison, or a juvenile correctional facility for more than $72 \mathrm{~h}$ in the preceding 12 months

5. People who have been newly diagnosed with or have been treated for syphilis, gonorrhea, chlamydia, or genital ulcers in the preceding 12 months

6. People who have been on hemodialysis in the preceding 12 months (increased risk for recent $\mathrm{HCV}$ infection only) [30]

Recipients of organs from PHS high-risk donors typically receive separate counseling about the potentially increased risk of viral transmission. All transplant recipients undergo pre-transplant testing for HIV, HBV, and HCV. Posttransplant serologic surveillance within the first year after transplant is recommended to exclude new infections in recipients of organs from PHS high-risk donors.

While patients with positive hepatitis B surface antigen are excluded from donation, selected patients with isolated positive hepatitis $\mathrm{B}$ core antibody $(\mathrm{HBcAb}+)$ may be considered as suitable donors. Initial evaluation requires excluding underlying hepatic fibrosis in case the donor had prior hepatitis B infection. If the graft is found to be suitable for donation, there is still a risk of viral transmission to the recipient. Risk of $\mathrm{HBV}$ infection in the recipient depends largely on whether the recipient has immunity against HBV. Specifically, although infection rates are low in hepa- 
titis B surface antibody ( $\mathrm{HBs} A b)$ positive/ $\mathrm{HBc} A b+$ recipients, they may be as high as $76 \%$ in recipients with both negative $\mathrm{HBsAb}$ and $\mathrm{HBcAb}$ [31]. As such, the recipient of a liver allograft from a $\mathrm{HBcAb}+$ donor will usually receive oral antiviral medication with or without hepatitis B immune globulin (HBIG) for prophylaxis. The use of lamivudine for prophylaxis has reduced the risk of infection to less than $4 \%$, and the use of newer agents such as tenofovir and entecavir with lower rates of resistance may result in even lower infection rates among OLT recipients from isolated $\mathrm{HBcAb}+$ donors. The addition of HBIG to oral antiviral agents may not provide additional benefit in this setting [32].

$\mathrm{HCV}$-seropositive (+) donor livers are used in HCVseropositive recipients. Assuming the absence of significant hepatic fibrosis in the donor liver, the use of $\mathrm{HCV}+$ donors for $\mathrm{HCV}+$ recipients can be considered and does not appear to affect patient survival, graft survival, or severity of HCV recurrence following transplantation [33].

Experience with HIV-seropositive (+) donors in organ transplantation is limited at this time. In 2013, the USA enacted the HIV Organ Policy Equity Act which allowed for the transplantation of HIV+ organs into HIV+ recipients. Initial reports of liver transplantation with HIV+ donors suggest good short-term outcomes.

\section{Surgical Approaches to Liver Transplantation}

The abdomen is typically opened with a bilateral subcostal incision with midline extension. To begin the recipient hepatectomy, the falciform and gastrohepatic ligaments are divided. The liver can then be lifted to expose the porta hepatis. Subsequently the hepatic artery, bile duct, and portal vein are sequentially divided. The patient is often placed on veno-venous bypass during this time. The suprahepatic and infrahepatic vena cava are clamped, and the recipient's liver is removed en bloc with the excluded portion of the vena cava. The gallbladder is removed along with the liver. Backtable preparation of the allograft typically occurs during the recipient hepatectomy. The allograft is then placed into the recipient, and the vena cava and portal venous anastomoses are created. The allograft is reperfused, and systemic bypass is discontinued. After reperfusion of the allograft, the arterial and biliary anastomoses are completed.

In certain cases, variant anatomy may require alteration in the creation of vascular or biliary anastomoses. An arterial conduit can be used if the recipient hepatic artery is insufficient. Roux-en-Y hepaticojejunostomy is considered in select cases such as when the recipient common duct is diminutive or large biliary collateral veins are present. Patients with primary sclerosing cholangitis typically require Roux-en-Y hepaticojejunostomy. In the past, $\mathrm{T}$ tubes were used in the case of donor-recipient duct size mismatch. However, their use has declined significantly in recent years due to the risk of complications including bile leak. Many centers will now consider closing a portion of the larger duct with suture prior to creating the biliary anastomosis.

An alternative to vena cava exclusion is the piggyback technique. In this situation, the hepatic artery, common duct, and portal vein are divided in the typical fashion. However, instead of clamping the vena cava and removing the liver en bloc with the vena cava, the surgeon instead dissects the liver surface off of the vena cava. The hepatic veins are clamped and divided, and the recipient liver is removed without disrupting the recipient vena cava. Longitudinal incisions are made in the recipient and donor vena cavas to allow for creation of a cavo-caval anastomosis. Potential benefits of the piggyback technique include decreased warm ischemia time, reduced blood transfusions requirements, and less need for veno-venous bypass. Piggyback reconstruction may increase the risk of developing hepatic venous outflow obstruction and subsequent Budd-Chiari syndrome.

\section{Surgical Complications Resulting in Infection}

Multiple surgical complications after LT may predispose to the development of infection. Hepatic artery thrombosis (HAT) is the most common vascular complication after OLT, with a $4 \%$ incidence reported in adult recipients [34]. HAT can be classified as early or late occurring before or after 4 weeks following transplantation. Early HAT may present in three distinct ways. The most severe presentation is fulminant hepatic failure, which is marked by acute rise in liver enzymes, encephalopathy, coagulopathy, and often sepsis. Other patients with HAT can present subacutely with ischemic injury to the bile ducts which predominantly rely on perfusion by the hepatic artery. These patients will subsequently develop biliary complications including strictures, acute cholangitis, hepatic abscesses, or recurrent episodes of bacteremia. A final group of patients with HAT may be asymptomatic and diagnosed incidentally.

HAT can be identified on Doppler ultrasound, although either radiographic or conventional angiography is often required to confirm the diagnosis. Patients with HAT and fulminant hepatic failure require management of sepsis including broad-spectrum antibiotics and prompt retransplantation. Less symptomatic patients can be taken to the operating room for hepatic artery revision and possible thrombectomy or managed non-operatively with catheter-directed thrombolysis. Patients who present with biliary complications of late HAT typically will develop a progressive course marked by recurrent cholangitis and other complications of biliary obstruction. In the short term, they can be managed with endoscopic or percutaneous biliary drainage and antibiot- 
ics during episodes of acute cholangitis. However, many of these patients will ultimately require retransplantation.

Biliary complications occur frequently with an average incidence of up to $25 \%$ reported in some series. Many types of complications may occur including anastomotic and nonanastomotic biliary strictures, bile leak, biliary abscess, obstruction due to stones or casts, or acute cholangitis. The majority of biliary complications occur in the first 3 months after transplant, although biliary strictures may present several years postLT. Approximately $80 \%$ of biliary strictures are at the site of anastomosis, but later presentations of non-anastomotic strictures may occur as a consequence of DCD organ use, older donor age, technical factors at the time of arterial anastomosis, HAT, hepatic artery stenosis, prolonged cold ischemia time, CMV infection, ABO blood group mismatch, or recurrent primary sclerosing cholangitis [35-37]. LDLT and split liver recipients are at increased risk for biliary complications as compared to DDLT recipients due to the cut surface of the liver and potentially more delicate biliary anastomoses. Most biliary structures are managed with endoscopic retrograde cholangiopancreatography (ERCP) or percutaneous biliary drainage. In patients with persistent anastomotic strictures despite repeated endoscopic therapy, surgical revision may be considered.

Intra-abdominal hemorrhage can occur in the early posttransplant period with a reported prevalence as high as $20 \%$. Preexisting coagulopathy and diminished hepatic synthetic function are associated with a higher risk of bleeding. Initial management is typically supportive, but reoperation may be required in up to $15 \%$ of cases if bleeding persists. However, a source of hemorrhage may be identified in only half of such cases [38].

\section{Immunosuppressive Regimens in Liver Transplantation}

The alloimmune response after LT is primarily T-cell mediated. The role of donor-specific antibodies and the concept of antibody-mediated rejection require further study but generally appear to be rare. Currently, induction immunosuppression with T-cell-depleting agents, such as thymoglobulin, is not used routinely after OLT. On Day 1, liver transplant recipients receive 500-1000 $\mathrm{mg}$ of methylprednisolone followed by a taper and conversion to prednisone. Initially, recipients are maintained on a multidrug regimen including a calcineurin inhibitor (CNI) such as tacrolimus, an antimetabolite agent such as mycophenolate, and corticosteroids.

CNIs serve as the backbone of immunosuppression regimens for most patients after liver transplantation. Most centers use tacrolimus as the preferred CNI. Direct comparisons between tacrolimus and cyclosporine after OLT have revealed lower rates of acute rejection and improved patient and graft survival with the use of tacrolimus [39-41]. CNIs are associated with nephrotoxicity, neurotoxicity, hypertension, and hyperlipidemia. Alopecia and hyperglycemia are more often seen with tacrolimus use, while hirsutism and gingival hyperplasia are more commonly associated with cyclosporine. Patients with acute liver failure and decompensated cirrhosis with severe hepatic encephalopathy may be more prone to neurotoxicity from these agents. As such, introduction of the CNI may be delayed until the patient is awake. CNIs are metabolized by the cytochrome p450 system; therefore, their serum concentration may be affected by several other commonly used medications. CNI levels and drug exposure will decrease with concurrent use of certain antiepileptic medications, rifampin, alcohol, and St. John wort. In contrast, CNI levels and drug exposure will increase with the use of macrolide antibiotics, azole and triazolebased antifungals, verapamil, and grapefruit juice.

In selected patients, mTOR inhibitors such as sirolimus or everolimus may also be used, often to minimize potential toxicities from a CNI-based regimen. Everolimus is FDA approved for use with a low-dose CNI in patients after liver transplantation, while sirolimus is not approved by FDA for use in patients undergoing liver transplants, and occasionally used off-label in select patients. The use of mTOR inhibitors within the first 30 days after liver transplant is discouraged due to the risk of hepatic artery thrombosis and impaired wound healing. Some studies suggest that early initiation of mTOR inhibitors within 90 days of OLT may lead to increased preservation of renal function [42-44]. The recommendation for concurrent use of a low-dose CNI is based on a higher risk of acute rejection for patients on mTOR monotherapy. Additionally, mTOR inhibitors do exhibit some antineoplastic activity and have been shown to reduce the risk of recurrent cutaneous squamous cell carcinoma after renal transplantation [45]. It is not clear that mTOR inhibitors improve long-term post-transplant recurrence risk in regard to HCC $[46,47]$. Besides the risk of hepatic artery thrombosis and impaired wound healing, other common adverse effects associated with mTOR inhibitors include oral ulcers, edema, proteinuria, hyperlipidemia, and bone marrow suppression.

Mycophenolate, an antimetabolite agent, is commonly used in conjunction with a CNI in patients after liver transplantation and allows for more rapid discontinuation of corticosteroids. In many patients, mycophenolate is withdrawn within the first year after transplant; however, prolonged use may be indicated in order to minimize adverse effects of CNIs, such as nephrotoxicity [48]. Additionally, some centers maintain patients transplanted for autoimmune liver diseases on mycophenolate for a longer duration given the increased risk of acute rejection or recurrent autoimmune disease after OLT. The use of mycophenolate in pregnancy is contraindicated based on an increased risk of pregnancy loss and congenital malformations. Azathioprine may be a substitute for mycophenolate at some centers. 
Glucocorticoids, usually prednisone, have multiple inhibitory effects on hosts' immune responses mediated by both innate and adaptive $\mathrm{T}$ and $\mathrm{B}$ cells. Due to their numerous side effects, most centers attempt to rapidly taper and wean patients off prednisone as soon as possible, often within the first 6 months after transplantation. Long-term low-dose prednisone has been utilized in patients with pre-transplant autoimmune liver disease. Since steroids increase HCV replication, abrupt changes in steroids, such as rapid withdrawal or bolus dosing for rejection, may be associated with worse outcomes [49].

Humanized monoclonal antibodies against the IL-2 receptor can be used in order to delay initiation of a CNI, particularly to avoid the immediate risk of nephrotoxicity or neurotoxicity. The use of daclizumab has demonstrated low rejection rates and improved renal function without an increase in CMV or other infections [50]. In 2009, daclizumab was removed from the market for commercial reasons. Basiliximab is commercially available and occasionally used clinically, although published data regarding the use of this immune modulator in liver transplant recipients are limited.

\section{Management of Rejection in Liver Transplantation}

Acute cellular rejection (ACR) occurs in up to $25 \%$ of OLT recipients, with the majority of cases occurring within the first month after transplant [51]. Antibody-mediated rejection is thought to be an uncommon phenomenon in this solid organ allograft transplant population. The risk of ACR after 1 -year post-transplant declines to $10 \%$ and is potentially associated with poor compliance with antirejection medication. Early ACR does not seem to affect graft survival, but late ACR has been associated with an increased risk of developing chronic rejection and graft loss [52]. In many cases, the initial suspicion for ACR arises when elevated liver enzymes are discovered. Symptoms including fever or jaundice are less common, particularly if ACR is identified early. Liver biopsy is required to make the diagnosis of ACR, which is defined by a triad of mixed cell portal inflammation, endotheliitis, and ductulitis [53]. The majority of cases of ACR can be managed with corticosteroid therapy. Typically a high dose of methylprednisolone $500-1000 \mathrm{mg}$ daily is given intravenously for 1-3 days with a subsequent taper. Patients with refractory ACR can be managed with either a second course of steroids or less often thymoglobulin. Thymoglobulin, a polyclonal antibody preparation directed against lymphocytes, is administered intravenously for up to 5 days with careful monitoring of white blood cell and platelet counts. All patients generally receive oral antimicrobial prophylaxis for CMV and Pneumocystis jirovecii when treated for ACR.
Chronic rejection (CR) occurs in less than $5 \%$ of OLT recipients in the modern era. Prior ACR, autoimmune liver disease, and CMV infection all may predispose to development of CR. Patients with CR typically present with laboratory evidence of cholestasis and may be jaundiced. As opposed to in ACR, steroid therapy is not beneficial in the management of CR. Options for management include increasing the dose of tacrolimus or the use of additional immunosuppressants such as mycophenolate mofetil or an mTOR inhibitor. Despite maximal medical therapy, many patients with $\mathrm{CR}$ will ultimately require retransplantation [54].

\section{Infectious Complications in Patients with Chronic Liver Disease and Cirrhosis}

Cirrhosis represents a state of progressive hepatic fibrosis with marked distortion of the hepatic architecture and development of regenerative nodules. The liver plays an important role in the hosts' immunity against bacterial pathogens; therefore, the development of chronic liver disease and cirrhosis renders such patients to an increased risk for infections. Thus, liver transplant candidates represent a unique patient population that is highly vulnerable to multiple infectious complications affecting the pre-transplant course. In addition, chronic liver disease and cirrhosis are associated with increased morbidity and mortality, due to gastrointestinal and variceal bleeding, severe and recurring ascites, hepatic encephalopathy, hepatorenal and hepatopulmonary syndromes, bile secretion impairment, and severe coagulopathy. Infections play an important role in the overall morbidity and prognosis for persons with chronic liver disease including cirrhosis and may contribute to $30-50 \%$ mortality [55]. Bacterial infections are noted at the time of hospitalization or during the course of hospital stay in $25-35 \%$ of patients with cirrhosis [56]. It is estimated that there is a $15 \%$ hospital mortality for cirrhotic patients who develop an infection episode [55]. In patients with cirrhosis, it is estimated that $30 \%$ are community-acquired infection, and the remainder are healthcare-onset infections. Approximately $35-40 \%$ of these healthcare-acquired infections will occur $>48 \mathrm{~h}$ after hospital admission, which places these patients at an increased risk for infections due to multidrug-resistant organisms (MDROs) [56, 57].

\section{Overview of Liver Function and Its Contribution to Host Defense}

The liver is an organ that plays an important role in the metabolism, synthesis, and the storage of nutrients and proteins. The liver produces the majority of the body's proteins and therefore has pivotal synthetic functions such as metab- 
olism and synthesis of amino acids, carbohydrates, fatty acids, lipoproteins, plasma proteins like albumin, transport proteins, protease inhibitors, fibrinogen, clotting factors, and complements. The liver also serves as a center of detoxification, removing or degrading toxic components from the circulation. In order for the liver to accomplish these functions, the hepatocyte must extract nutrients, waste, and toxic products from the blood that circulate through the liver within its sinusoids. The liver is continuously exposed to dietary ingredients consisting of exogenous molecules and micronutrients, environmental products, and byproducts of the gastrointestinal microbiota. The blood from the gastrointestinal and mesenteric circulation enters the liver via the portal vein and mixes with oxygen-rich blood from the systemic circulation via the hepatic artery and ultimately drains into the liver sinusoids. It is estimated that $80 \%$ of the hepatic blood flow within the liver arises from the gastrointestinal tract through the portal circulation [58]. Therefore, hepatocytes are exposed to a mixture of portal venous and arterial blood. The sinusoids are lined with liver sinusoidal endothelial cells that contain fenestrations to facilitate passage of the blood to reach the hepatocytes [58]. The hepatocytes are constantly exposed to foreign and immunogenic antigens and environmental toxins and those produced by the endogenous orointestinal microbiota. This constant exposure could result in untamed systemic immune activation; however, the liver plays a critical role in regulation and maintenance of immunologic and inflammatory homeostasis [58].

In addition to metabolism and synthesis, the hepatocytes play an important role in host defense. Their contribution to host immunity includes the production of complement and antimicrobial proteins, acute phase proteins in response to infection, and antigen presentation to T cells [58]. The liver is also a lymphoid organ with unique immunological properties. The liver-specific macrophages, known as Kupffer cells, line the luminal surface of the hepatic sinusoids and are an important part of the reticuloendothelial system (RES). The Kupffer cells in the liver comprise $90 \%$ of the body's tissue macrophages and make up one-third of the parenchymal cells in the liver [59]. Both the Kupffer cells and the hepatocytes contain pattern recognition receptors (PRR) which can bind to microbe-associated molecular patterns and damageassociated molecular patterns that originate from the gastrointestinal tract through the portal circulation [60-62]. These molecular patterns are then phagocytized by the Kupffer cells or hepatocytes and are removed and cleared from the circulation. These local processes of removal and degradation by the Kupffer cells and the hepatocytes serve a protective role to prevent systemic immune activation as a result of antigens and byproducts from the gastrointestinal tract [58]. These properties ensure an efficient innate defense against intestinal organisms and toxins and confer a particular capacity for preservation of immune tolerance. The normal liver is therefore considered to be immune tolerant or "tolerogenic." This is best supported by the observations that liver transplant recipients can reduce and also wean their immunosuppressive therapy in up to $20 \%$ of the patients, compared to recipients of other solid organ transplant [63].

On the other hand, with regard to host defense, the Kupffer cells of the liver play a key role in the removal of bacteria and their endotoxins from the bloodstream, as well as producing local inflammatory cytokines. Their presence within the vascular sinusoids provides an effective first line of defense against infections via the hematogenous route and bacterial translocation from the gastrointestinal tract.

\section{Immunologic Dysfunction in Cirrhosis}

In patients with cirrhosis, this crucial hepatic immunologic homeostasis is severely disrupted. Additionally, a milieu of dysregulated systemic pro-inflammatory cytokine response can result in further organ damage, especially in cirrhotic patients with systemic infection or sepsis [57]. The immunopathogenesis seen in cirrhosis is quite complex and is outlined in Fig. 3.1. In general, the clearance of bacteria and bacterial endotoxins by the Kupffer cells is impaired in patients with cirrhosis due to portosystemic shunting. The immunologic dysfunction associated with cirrhosis is further augmented by compromised liver synthetic function, malnutrition, stress catabolism, and also lifestyle factors, such as alcohol consumption. Chronic liver disease and cirrhosis are associated with alterations and/or deficiencies in all of the host defense mechanisms. In addition to depressed host immunity, these patients are at increased risk for exposure to healthcare-acquired infections due to their frequent need for hospitalization to manage the many complications associated with chronic and end-stage liver disease.

The key host factors that contribute to an increased risk of infection in patients with cirrhosis include (1) alterations in the intestinal microbiota and the intestinal mucosal barrier; (2) depression of activity by Kupffer cells and RES; (3) suboptimum opsonic activity in serum and the ascitic fluid; (4) neutrophil dysfunction with decreased phagocytosis and chemotaxis; and (5) decreased production and abnormalities in complement pathways, pattern recognition receptors, and C-reactive protein [57].

In recent years there has been an increased appreciation of the importance of the normal gut microbiota which contributes to both the host's metabolic and immunologic functions. The majority of the gut microbiota is comprised of obligate anaerobes within the phylum Firmicutes (specifically Clostridia spp. and Gram-negative anaerobes such as the Bacteroides spp.). In the bowel of healthy subjects, Gram-negative bacteria are present in relatively low numbers as compared with the obligate anaerobes, whereas 


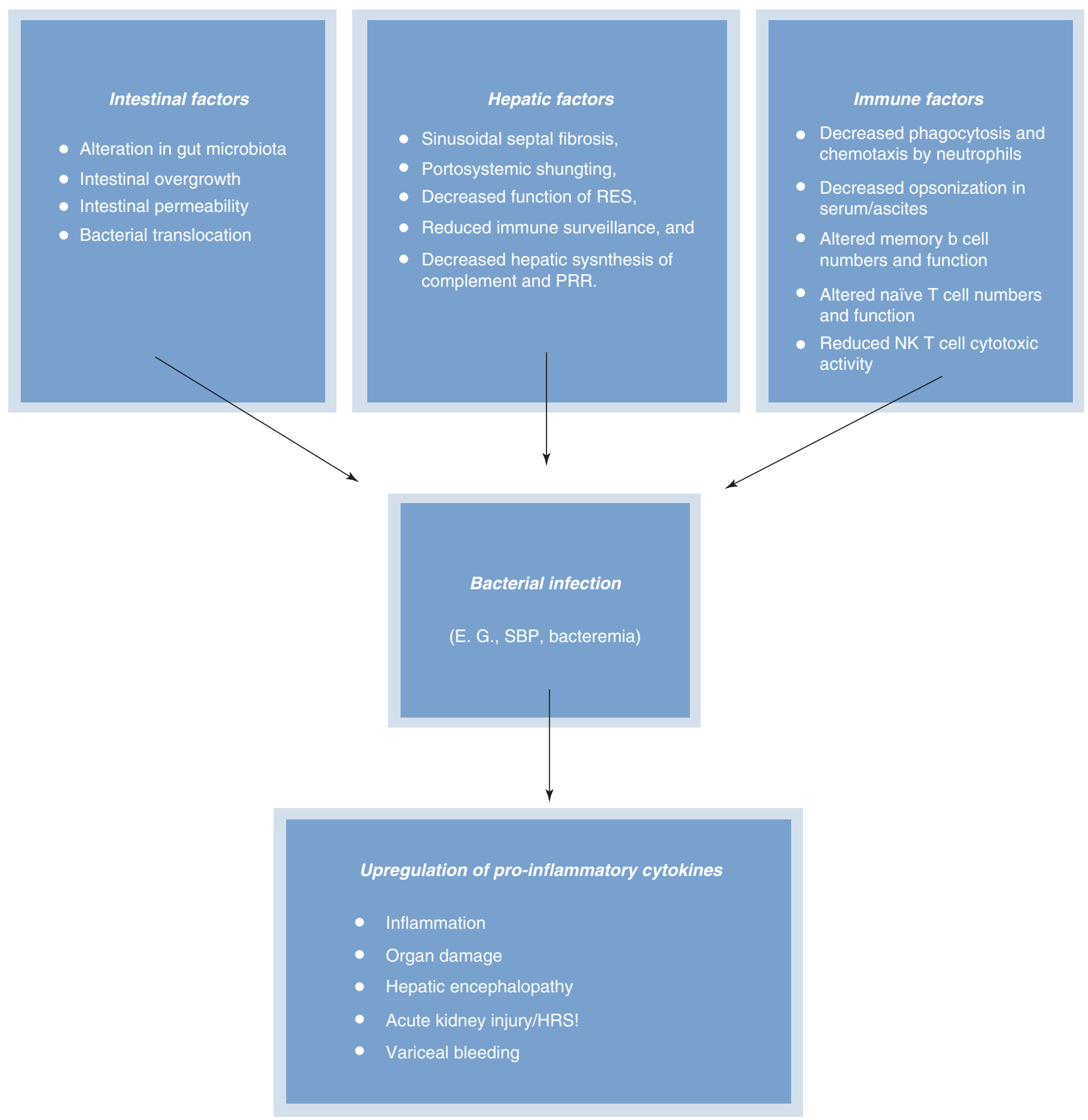

Fig. 3.1 Immunologic dysfunction associated with cirrhosis. PRR, pattern recognision receptors. SBP, spontaneous bacterial peritonitis. HRS, hepato-renal syndrome

with cirrhosis, the proportion of Gram-negative bacteria is more prominent. Alterations in the composition of the gut microbiota are well recognized in patients with cirrhosis, with an increase in colonization by the Proteobacteria that are predominantly Gram-negative enteric bacteria [64]. Spontaneous infections with Gram-negative bacilli are common in patients with cirrhosis, and it is proposed that this is mainly due to bacterial translocation from the gas- trointestinal tract that has undergone alteration in the gut microbiota. Bacterial translocation occurs when bacteria or yeast migrate to the mesenteric lymph nodes and into the portosystemic circulation [57]. In animal experiments, oral administration of radiolabeled E. coli was detected within the intestinal lumen, mesenteric lymph nodes, and the ascitic fluid in mice with cirrhosis [65], supporting the possible mechanism of enhanced bacterial translocation 
that may also occur in patients with cirrhosis. Additionally, Gram-negative bacilli are demonstrated to be more efficient in translocating across bowel lumen when compared to obligate anaerobes in a murine experimental model [66], providing further evidence for clinical observations that Gram-negative enteric bacilli are the most common cause of infection in patients with chronic end-stage liver disease and systemic infections due to anaerobes are seldom seen. Other factors that contribute to the bacterial translocation of pathogenic bacteria in cirrhosis include intestinal bacterial overgrowth and increased intestinal permeability [64]. Intestinal overgrowth and alterations in the gut microbiota appear to correlate with the Child-Pugh score; higher prevalence of bacterial overgrowth has been observed in patients with Child-Pugh classes B and C compared with patients having class A liver disease [67, 68]. Moreover, intestinal bacterial overgrowth has been associated with a diagnosis of minimal hepatic encephalopathy and supports the use of nonabsorbable rifaximin in the treatment and prevention of hepatic encephalopathy in such patients [68]. It has also been shown that there is a higher bacterial burden of pathogenic E. coli in stool cultures in patients with cirrhosis and those with early-stage hepatic encephalopathy [69]. Normal hosts have tight junctions between mucosal and epithelial cells, which limit translocation of bacteria and bacterial products. Patients with cirrhosis have alterations in tight junction or desmosome proteins that compromise the physiologic barrier and may result in increased bacterial translocation [70, 71]. Additionally, intestinal bacterial access to the gut epithelial cells may be facilitated by deficiencies in IgA, bile lipids, and antimicrobial peptides that are notably observed in patients with advanced cirrhosis $[64,72,73]$. Therefore, changes in the gut microbiota, coupled with the increased intestinal permeability, particularly in cirrhotic patients with ascites greatly enhance the risk for bacterial translocation due to aerobic Gram-negative bacteria resulting in spontaneous bacterial peritonitis and bloodstream infections.

Cirrhosis is associated with both sinusoidal and septal fibrosis which results in portosystemic shunting. Patients with cirrhosis have depressed RES-Kupffer cell function, which can further promote translocation of bacterial pathogens and their endotoxins, thus reducing immune surveillance by the Kupffer cells/RES and allowing these pathogens access to the bloodstream [74]. Toll-like receptors (TLRs) are expressed on the surface of macrophages including Kupffer cells and can recognize and bind to bacterial products including endotoxins. Alterations in the TLRs and nucleotide-binding oligomerization domain (NOD) 2 gene via minor genetic polymorphisms may result in decreased affinity of TLR for Gram-negative bacilli lipopolysaccharide further enhancing the risk for bacterial infections in patients with cirrhosis [75, 76]. Furthermore, it has been demonstrated that simultaneous variations in the NOD 2 and TLR genes are associated with increased risk for spontaneous bacterial peritonitis, as well as an increase in surrogate markers for intestinal permeability in patients with cirrhosis [77]. It appears that genetic polymorphisms may contribute to the risk of spontaneous bacterial peritonitis and other bacterial infections in patients with chronic liver disease and cirrhosis.

Patients with cirrhosis experience both generalized immunodeficiency and systemic immune activation with the production of pro-inflammatory cytokines, also referred to as cirrhosis-associated immune dysfunction (CAID) [77]. There are numerous immunologic deficits that contribute to CAID and ultimately risk of systemic infection. In patients with cirrhosis, there is an overall reduction of circulating immune cells, and this is most notable for neutrophils, naïve $\mathrm{T}$ cells, and memory $\mathrm{B}$ cells [78]. In addition to reduced numbers of immune cells, there is a cellular dysfunction, including reduced phagocytic and chemotactic properties of neutrophils, reduced phagocytic activity of monocytes, hypoproliferative response to mitogens by $\mathrm{T}$ and $\mathrm{B}$ cells, and reduced natural killer cell cytotoxic activity [79-82]. Stunted TNF $\alpha$ production and HLA-DR expression have also been noted in cirrhotic patients with acute decompensation, such as sepsis, and referred to as "immune paralysis" [83].

Finally, as cirrhosis affects the synthetic function of the liver, the production of both complement and pattern recognition receptors (PRRs) are diminished [84]. Several important soluble PRRs produced by the liver include C-reactive protein, lipopolysaccharide-binding protein, peptidoglycan recognition protein, and soluble CD14, which activate complement associated opsonization cascade $[85,86]$. Reduction in complement and PRR synthesis decreases bactericidal function of phagocytic cells in patients with cirrhosis [87]. Specifically, decreased concentrations of C3, C4, and CH50 result in suboptimal opsonic activity in both serum and ascitic fluid and have been recognized as an important risk factor that is associated with increased susceptibility to bacterial infections [88, 89]. Bactericidal functions of neutrophils are compromised resulting from decreased circulating cells in the peripheral blood due to splenic sequestration, decreased chemotaxis, and phagocytosis [88]. In patients with cirrhosis, neutrophils have reduced microbicidal activity owing to diminished intracellular superoxide production and myeloperoxidase activity [90]. Additionally, neutrophil dysfunction can be further exacerbated by alcohol consumption further suppressing phagocytosis and increasing the risk of bacterial infection [91].

In summary, the immunologic and synthetic function of the liver plays an important role in immune surveillance and immunologic homeostasis. Patients with advanced liver disease awaiting liver transplantation are a vulnerable population due to hepatic structural abnormalities, reduced production of critical immuno-protective proteins, reduced circulating immune cells, and impaired function of these 
cells. This overwhelming acquired immunodeficiency in patients with end-stage liver disease is further augmented by the frequent exposure to the healthcare setting, placing these patients at risk for numerous infectious complications prior to undergoing hepatic allograft transplantation.

\section{Clinical Aspects of Infections in Patients with Chronic Liver Disease and Cirrhosis}

As a result of CAID, infectious complications are extremely common in patients with chronic liver disease and may be the cause of mortality in up to $50 \%$ of these patients [9294]. The most common types of infections are spontaneous bacterial peritonitis (SBP), UTI, pneumonia, bloodstream infections, and skin and soft tissue infections [95, 96]. The bacteriologic causes of these infections are predominantly ( $75 \%$ ) due to Gram-negative bacilli (GNB); Grampositive organisms and anaerobes account for $20 \%$ and $3 \%$, respectively [97]. It is estimated that $64 \%$ of these infections may be due to drug-resistant bacteria [98]. Infection in patients with cirrhosis can exacerbate liver failure and precipitate end-organ damage at other sites [99]. Due to the dysregulation of the pro-inflammatory cytokine response in patients with cirrhosis, sepsis carries a mortality rate of $26-44 \%$. Infection can cause acute decompensation in individuals with chronic liver failure, which can result in worsening hepatic encephalopathy. Acute kidney injury with hepatorenal syndrome, acute lung injury with ARDS, and severe coagulopathy with gastrointestinal bleeding can all be precipitated by bacterial infections in patients with cirrhosis [62].

Spontaneous bacterial peritonitis is a common infection in cirrhotic patients with ascites. Severe, potentially life-threatening SBP that requires hospitalization often noted in patients with advanced liver disease may be associated with $31 \%$ mortality [59]. SBP is characterized by the spontaneous infection of ascitic fluid in the absence of an intra-abdominal source of infection. The pathogenesis of SBP is caused by bacterial translocation from the intestinal tract in the setting of portosystemic shunting. The most common organism isolated from peritoneal fluid is Escherichia coli. In a large series of 519 patients with SBP, the prevalence of culture-positive organisms included $E$. coli (43\%), Klebsiella pneumoniae (11\%), Streptococcus pneumoniae (9\%), other streptococcal species (19\%), other Enterobacteriaceae (4\%), Staphylococcus species (3\%), and Pseudomonas aeruginosa (1\%), and $10 \%$ were miscellaneous organisms [100]. Another study noted that in the setting of norfloxacin prophylaxis for SBP, viridans group streptococci were prominent streptococcal isolates followed by group B Streptococcus, Streptococcus pneumoniae, and Streptococcus bovis, emphasizing the increasing importance of streptococcal species breakthrough infection as a cause of SBP in patients receiving fluoroquinolone prophylaxis [101]. Guidelines and recommendations for the diagnosis, management, and prevention of SBP have been published and are updated by the American Association for the Study of Liver Diseases (AASLD) [102]. After SBP, UTIs are the next common site of infection in patients with cirrhosis. As in other patient populations, urinary catheters are an important risk factor for such infections, and as expected, GNB are common causative pathogens.

Patients with cirrhosis are at increased risk for communityacquired pneumonia (CAP), healthcare-acquired pneumonia (HCAP), and aspiration pneumonia. As patients with cirrhosis have both complement and B-cell defects, they are at increased risk for infections due to encapsulated organisms such as Streptococcus pneumoniae, Haemophilus influenzae, and Klebsiella pneumoniae. Additionally, these patients are more likely to have concurrent bacteremia and have multilobar involvement compared with CAP in the general population [103]. The depressed cellular immune response associated with cirrhosis also places these patients at increased risk for legionellosis. These patients require frequent hospitalization, and therefore they are at increased risk for HCAP. Hepatic encephalopathy is a risk factor for HCAP, because these patients have decreased mental status and are at risk for aspiration of oropharyngeal contents that often are colonized by Gram-negative enteric bacilli. Empiric treatment for HCAP should include coverage for Pseudomonas aeruginosa and other drug-resistant GNB, as well as MRSA. Patients with cirrhosis are at increased risk for aspiration events in the setting of altered mental status due to hepatic encephalopathy or excessive alcohol consumption, which places them at risk for lower respiratory tract infections [104]. Additionally, the periodontal disease that is seen in alcoholic patients provides a favorable environment for both anaerobes and Klebsiella pneumoniae colonization, which can lead to lower respiratory tract infection during aspiration events $[67,105]$. It is well recognized that patients with chronic alcoholism are at an increased risk for community-acquired pneumonia due to S. pneumoniae; a study reported that alcohol abuse was also a risk factor for CAP and septic shock due to Pseudomonas aeruginosa and Acinetobacter species [106]. It is important to note that the latter two infections are uncommon in patients with alcoholic cirrhosis unless these patients have extensive prior healthcare exposure including stays in the intensive care unit or need for mechanical ventilatory support; other risk factors include leukopenia or neutropenia.

Bloodstream infections can occur as a result of bacterial translocation from the gastrointestinal tract; Gram-negative enteric bacilli, enterococci, and Streptococcus spp. are common pathogens [58, 107]. It has been reported that bacteremia or SBP can complicate gastrointestinal bleeding in $17-45 \%$ of the episodes [108]. The management of esopha- 
geal varices involves esophageal variceal ligation (EVL) and esophageal variceal sclerotherapy (EVS); both of these procedures may increase the risk for transient bacteremia. In a meta-analysis, the overall rate of bacteremia following these procedures was $13 \%$; bacteremia was significantly more common after EVS (17\%) compared with EVL (6\%) [109]. The most common cause of transient bacteremia associated with EVS and EVL includes alpha-hemolytic Streptococcus and coagulase-negative staphylococci $[110,111]$. As with other hospitalized patients, invasive procedures such as indwelling central venous catheters place these patients at increased risk for bloodstream infections due to Staphylococcus aureus including MRSA and coagulase-negative staphylococci. Presence of cirrhosis was noted as an independent risk factor for the development of spontaneous bacteremia due to group B Streptococcus [112].

Hepatic hydrothorax is another complication of cirrhosis, and this can evolve into spontaneous bacterial empyema. Spontaneous bacterial empyema may be seen in 10-20\% of patients with hepatic hydrothorax, and like SBP, these infections are primarily due to Gram-negative enteric bacilli [113-115].

Skin and soft tissue infections including necrotizing fasciitis have been reported in patients with cirrhosis. In patients with end-stage liver disease, generalized edema, especially involving the lower extremities, is not uncommon; furthermore, suboptimal personal hygiene and malnutrition place these patients at increased risk for relapsing skin and soft tissue infections. The common causative agents are Staphylococcus aureus, including CA- and HA-MRSA, and $\beta$-hemolytic Streptococcus including group B Streptococcus; GNB infections have also been reported [62]. Other rare causes of skin and soft tissue infections due to Vibrio vulnificus and Aeromonas hydrophila may be seen more often in patients with cirrhosis compared with the general population.

\section{Specific Pathogens}

Patients with chronic liver disease exhibit CAID due to decreased complement levels, less effective phagocytic activity and chemotaxis, "bypass" of the reticuloendothelial system due to portosystemic shunting, and availability of free iron that promotes growth of particular organisms.

Iron is bound to proteins such as hemoglobin, ferritin, transferrin, and lactoferrin, which maintain a low level of free iron that inhibits sustained bacterial growth. The liver plays a key role in iron metabolism, and this can be significantly disrupted in patients with chronic liver disease and cirrhosis. While this disruption in iron homeostasis is most notable in hemochromatosis, it has also been reported in patients with alcoholic liver disease, nonalcoholic fatty liver disease, and chronic hepatitis $\mathrm{C}$ infection. Cirrhosis is associated with decreased synthetic function, including the production of hepcidin, an important regulatory peptide for iron metabolism. In response to infection and inflammation, there is increased production of hepcidin, which decreases the availability of free iron, resulting in induced hypoferremia. The decreased levels of free iron by hepcidin have important antimicrobial properties, by creating an environment that does not support bacterial growth. Animal models of hepcidin-deficient mice showed increased susceptibility to infection with Vibrio vulnificus. Therefore, suggesting that reduced production of hepcidin in patients with chronic liver disease and cirrhosis contributes greatly to their susceptibility to specific pathogens [116-118].

Noteworthy pathogens that are more common in patients with chronic liver disease and cirrhosis, as compared to the general population, include E. coli, Vibrio spp., Aeromonas hydrophila, Plesiomonas shigelloides, Yersinia spp., Salmonella spp., Listeria monocytogenes, Mycobacterium tuberculosis, and the invasive molds like Aspergillus fumigatus and Rhizopus spp. These organisms are able to take advantage of the immunodeficiency associated with chronic liver disease and cirrhosis; additionally high free iron levels provide favorable growth environment for such organisms [62, 79, 119].

The Vibrio spp., including V. vulnificus, V. cholera, and $V$. parahaemolyticus, can cause severe infection in patients with chronic liver disease and those with cirrhosis. Vibrio vulnificus is a Gram-negative, halophilic noncholera Vibrio species that is a common organism isolated from estuarine and marine environments and has been associated with gastroenteritis, skin and soft tissue infections with hemorrhagic bullae, and bacteremia resulting in severe sepsis and death. Impaired iron metabolism and increased iron availability appear to underlie the pathogenicity of $V$. vulnificus invasive disease [120-122]. Common coastal sites where V. vulnificus is endemic include the Chesapeake Bay and the Gulf Coast. Most patients (90\%) with V. vulnificus bacteremia have a history of consuming raw or undercooked shellfish specifically oysters, whereas skin and soft tissue infections are associated with the handling of raw seafood or direct inoculation due to recreational exposure to marine environment [84, 123, 124]. Gastroenteritis is the most common clinical manifestation, cellulitis with hemorrhagic bullae, and less commonly seen necrotizing fasciitis has also been reported. Patients with chronic liver disease or cirrhosis can present with primary $V$. vulnificus bacteremia associated with multiple hemorrhagic bullae and septic shock. In case series, V. vulnificus bacteremia carries a mortality of $40 \%$, with an extremely poor prognosis in patients with septic shock at the time of presentation [83-86, 125]. Therefore, it is recommended that persons with chronic liver disease should avoid consuming raw undercooked shellfish and exposure to marine water. 
Aeromonas species are GNB that are isolated predominantly from fresh water. Aeromonas hydrophila is the most common species and mainly presents as gastroenteritis. A rapidly progressive cellulitis or necrotizing fasciitis may rarely occur in such patients following exposure to fresh water. Bacteremia due to Aeromonas spp. is more common in patients with underlying cirrhosis and was associated with a $36 \%$ crude mortality [126]. In the same series, Aeromonas bacteremia, cirrhosis was the common underlying disease. Aeromonas SBP is an uncommon illness that may be observed during the summer months and associated with a diarrheal illness [127]. Plesiomonas shigelloides is also a GNB which can be acquired by the ingestion of raw or undercooked shellfish. It has been associated with gastroenteritis and in rare instances SBP [128, 129]. It may have a propensity to cause severe disease in iron overload conditions such as hemochromatosis.

The Yersinia spp., Y. enterocolitica, and Y. pseudotuberculosis are Gram-negative enteric bacilli that are also ferrophilic. Therefore, patients with cirrhosis and hemochromatosis are at risk for these gastrointestinal pathogens, which can be transmitted by undercooked pork products. In rare instances, Yersinia enterocolitica bacteremia has been associated with blood transfusions [130].

Listeria monocytogenes are Gram-positive bacilli that may be acquired via the ingestion of infected processed and canned food products or unpasteurized milk and products made from raw milk such as certain variety of cheese. The most important risk factor for invasive L. monocytogenes infection is depressed cellular immunity; availability of free iron in the hosts has been recognized as an important determinant of Listeria pathogenicity and virulence. Hence L. monocytogenes infections have been described in patients with hemochromatosis and cirrhosis [131-133]. Bacteremia and meningitis are the common clinical presentations of $L$. monocytogenes invasive disease.

Infections due to Mycobacterium tuberculosis, Aspergillus fumigatus, and Rhizopus spp. can rarely complicate the course of patients with chronic liver disease and cirrhosis due to the underlying immunodeficiency coupled with the alteration in iron homeostasis $[62,79,80,82]$.

\section{Infectious Complications in Liver Transplant Recipients}

\section{Epidemiology}

The incidence of infection after liver transplantation (LT) is particularly high compared to other solid organ transplants, likely due to the complexity of the procedure, risk of abdominal contamination, and especially due to poor medical status of many allograft recipients with end-stage liver disease.
Although mortality has markedly improved post-LT (), it is estimated that up to two-thirds of all LT patients suffer at least one episode of infection [134].

The risk of infection post-transplant is primarily determined by epidemiologic exposures including donor-derived, recipient-derived, healthcare-acquired, and communityacquired pathogens - and the patient's net state of immunosuppression. The net state of immunosuppression is based on multiple factors and includes the level of pharmacologic immunosuppression, underlying host factors, surgical procedures and other medical interventions, and viral infections (such as CMV). This is a complex interaction, and it generally varies predictably over time, based on typical protocols for antirejection immunosuppressive therapy and prophylactic strategies. Consequently, the risk of infection post solid organ transplant is traditionally subdivided into three distinct intervals: from surgery to 1 month post-transplant, 1 month to 6-12 months post-transplant, and beyond 6-12 months post-transplant $[135,136]$.

\section{Pre-transplant Screening for Infections}

Pre-transplant screening of both potential donors and recipients can help prevent infectious complications in multiple ways: by identifying contraindications to transplant, by detecting latent or occult active infections, and by stratifying risk for future infections and allowing appropriate preventive steps such as prophylactic antimicrobials or vaccination. Historically, screening approaches have differed widely between organ procurement organizations within the USA and internationally $[137,138]$. With greater experience and ability to diagnose transplant-related infections, there is now an established subset of infections that are routinely screened for in most cases. Most transplant centers' screening protocols require and perform the following tests in donors and recipients prior to transplantation and include human immunodeficiency virus (HIV) antibody, HSV (herpes simplex) IgG antibody (at some centers), cytomegalovirus (CMV) IgG antibody, hepatitis $\mathrm{C}$ virus (HCV) antibody, hepatitis B virus (HBV) surface antigen (HBsAg), hepatitis B core antibody (HBcAb IgM and IgG, or total core antibody), hepatitis B surface antibody (HBsAb), rapid plasma reagin (RPR), toxoplasma antibody (especially in heart recipients), Epstein-Barr virus (EBV) antibody (EBV VCA $\operatorname{IgG}, \operatorname{IgM}$ ), varicella zoster virus $(\mathrm{VZV})$ antibody, purified protein derivative (PPD) or interferon gamma release assay (IGRA) for latent TB infection in recipients, Strongyloides serology (for recipients from endemic areas), Coccidioides serology (for recipients from endemic areas), Trypanosoma cruzi serology (for donors and recipients from endemic areas), serologies for tetanus, diphtheria, measles, mumps, and 
pneumococcal titers as an aid to pre-transplant immunization (at some centers).

Some optional screening measures include West Nile virus serology or NAT HHV-8 serology and BK serology in kidney donor and recipients. NAT for HIV, HCV, and HBV is also performed and is of increasing importance especially in donors with high-risk social behaviors.

However, screening practices can still vary by transplant center [139]. Caregivers may choose to expand screening tests based on epidemiological or clinical factors on a caseby-case basis. Presurgical testing for LT is generally similar to protocols for other solid organ transplants; however, addressing potential latent Mycobacterium tuberculosis infection (LTBI) in candidate recipients raises some specific challenges.

Immunocompromised states may confer a weakened ability to react to tuberculin skin tests (TSTs) and interferon gamma release assays (IGRAs), decreasing the sensitivity of these tests when screening for Mycobacterium tuberculosis (MTb) [140, 141]. Individuals with cirrhosis are known to have impaired immune responses, including altered T-cell-dependent functions, which may affect the sensitivity of MTb testing [142]. There is no gold standard for latent MTb diagnosis, and all current tests have questionable accuracy for immunocompromised hosts. However, they do have high specificity and, thus, are still a valuable tool for minimizing the risk of active tuberculosis infection for many patients after undergoing transplantation [143-145]. Although there is limited research on the use of these tests specifically in individuals prior to transplantation, 2013 guidelines from the American Society of Transplantation recommend standard TST for all transplant candidates, with consideration of a second boosting TST 2 weeks later for those who initially test negative; this can potentially reveal a positive test for patients with remote exposure [146]. IGRAs are also recommended as an alternative to TST and may be preferable for convenience at most transplant centers. The use of an IGRA for screening for LTBI is favored in the setting where a patient has received a prior BCG vaccine, as an IGRA is more specific than a TST in this instance. The QuantiFERON-TB Gold In-Tube test (QFT) (Cellestis, Australia) has been compared to the TST for diagnosis of latent tuberculosis infection in candidates being considered for LT, and of note, indeterminate QFT results were more likely in patients with higher MELD scores [145]. There is some evidence that the T-SPOT TB test (Oxford Immunotec Ltd) may have slightly higher sensitivity than the QuantiFERON-Gold In-Tube test [147].

Treatment for LTBI also poses a unique challenge for pre-LT candidates, given the hepatotoxic potential of the treatment options. Completion of treatment before transplantation is ideal to best minimize the risk of developing active tuberculosis infection. With careful monitoring, even compensated cirrhotic individuals can complete the treatment safely [148, 149]. If exposure to MTb is suspected to be recent (i.e., if the candidate had recent conversion of their TST or IGRA from negative to positive), early treatment is especially favored, as risk for MTb activation is highest during the initial 1-2 years post exposure [150]. However, for candidates with advanced decompensated liver disease, it is usually advisable to defer treatment till after transplant when the patient is deemed stable from a liver function standpoint, to minimize the risk of fulminant hepatotoxicity.

There are two standard treatment options for LTBI in preliver transplant candidates: isoniazid for 9 months or rifampin for 4 months. The risk for hepatotoxicity has not been shown to be significantly different between the two drugs for this population [145]. Due to the contraindicated drug interactions between rifampin and many immunosuppressants, rifampin is not recommended following transplantation. The coadministration of rifampin with tacrolimus results in decreased drug exposure of tacrolimus. Twelve weeks of directly observed therapy with isoniazid and rifapentine is a third approved regimen for LTBI in the general population; however, as it has not been studied in the LT population, it is not recommended in these cases. Additionally, rifapentine is similar to rifampin in its effect on tacrolimus drug exposure. A randomized prospective study sought to investigate the efficacy and safety of 9 months of levofloxacin prophylaxis in LT candidates; however, the study was terminated early due to an $18 \%$ rate of severe tenosynovitis in the levofloxacin $\operatorname{arm}[151]$.

\section{Timeline for Infectious Complications from Transplant Procedure to 1 Month After Transplantation}

\section{Bacterial and Healthcare-Acquired Infections}

In the first month post-LT, infections are most often healthcare associated and bacterial in origin, similar to the infection risks for immunocompetent hosts undergoing other hepatobiliary procedures. A variety of factors are associated with an increased risk for bacterial infection. These factors include older age, length of preoperative stay, CMV infection, duration of surgery, retransplantation, volume of transfused blood products, preoperative MELD and CTP scores, bilioenteric anastomosis, technical complications (e.g., biliary leak, HAT), renal replacement therapy, and hyperglycemia [152]. Intra-abdominal infections are most common due to the technical nature of the surgery. The bile duct has no natural collaterals, being supplied only arterially, as opposed to the liver, which has dual circulation through the portal system. The biliary epithelium is thus more vulnerable to hypoperfusion during transplantation or resulting from hepatic 
artery thrombosis (HAT). A 30-year review at one center found a $14 \%$ rate of biliary complications post-LT, including anastomotic and non-anastomotic strictures, bile leaks, and cholangitis. Elevated MELD, HAT, and elevated donor creatinine suspected to reflect altered metabolic factors at the site of anastomosis, which may impair healing, were all significant risk factors for anastomotic biliary complications [153]. Biliary complication may in turn lead to further infectious complications, such as hepatic and intra-abdominal abscesses. A 10-year retrospective study at a large transplant center diagnosed hepatic abscess post-LT at a rate of 4.8 per 1000 transplant patient-years [154]. In the same study, HAT was confirmed to be a significant predisposing factor for hepatic abscess. Management of infectious biliary complications warrants antibiotic coverage for enteric Gram-negative organisms and anaerobes, as well as healthcare-associated bacteria based on the patient's history. Source control may necessitate drainage, endoscopic intervention, surgical repair or in severe cases, retransplantation.

After intra-abdominal infections, the lungs are the second most frequent site of infectious complication post-LT. A retrospective study of deceased donor hepatic allograft recipients found an incidence of 10 episodes of ventilatorassociated pneumonia per 1000 days of mechanical ventilation. Enterobacteriaceae accounted for $79 \%$ of bacterial etiologic agents. Both intra-abdominal infection and pneumonia can be complicated by concurrent bacteremia. A multicenter retrospective study found bloodstream infections complicated $29 \%$ of LT recipients in the first year after transplantation, with $52 \%$ of infections occurring within the first 100 days [155].

Surgical wound infections are third common infectious complications during this period, followed by urinary tract infections and other healthcare-associated infections (HAIs) [150]. Risk factors identified for surgical site infection include prolonged operative time, large-volume blood transfusion, biliary leak, retransplantation, dialysis, and CMV infection [156, 157].

Antibiotic resistance has become an increasing problem for treating HAIs. In 2014, the CDC found that $14 \%$ of HAIs in short-term acute care hospitals were caused by 1 of 6 major antibiotic-resistant threat bacteria [158]. The most recent survey of HAIs from the National Healthcare Safety Network from 2011 to 2014 further showed that resistance patterns continue to change over time [159]. Antibiotic resistance is particularly a concern for liver transplant recipients who are likely to have had prior antibiotic exposures as a result of numerous hospitalizations associated with the complications of chronic liver disease in the pre-transplant period. A study of 300 liver transplant recipients found 88 suffered at least 1 infection in the early 30-day post-transplant period; and of these, 78 (89\%) were due to drug-resistant bacteria [159]. The multidrug-resistant organisms (MDROs) most commonly encountered in LT recipients include extended $\beta$-lactamaseproducing Enterobacteriaceae (ESBL), MDR Pseudomonas aeruginosa, carbapenem-resistant Acinetobacter baumannii (CR-AB), carbapenem-resistant Enterobacteriaceae (CRE), vancomycin-resistant Enterococcus (VRE), and methicillinresistant Staphylococcus aureus (MRSA) [160].

The most common MDROs associated with ESBL production are Klebsiella pneumoniae and E. coli. Although it appears that ESBL-producing organisms may be more common in kidney transplant recipients, often as an etiology for UTIs, LT recipients are also at risk for these organisms and have been reported in 5.5-7\% [161-163]. In a large solid organ transplant cohort, $53 \%$ of the Klebsiella pneumoniae isolates were ESBL producing, and the highest risks were seen in kidney transplant recipients, especially those requiring post-transplant renal replacement therapy [162]. Infections due to ESBL-producing organisms in solid organ transplant recipients have been noted to be associated with mortality that ranged from $5 \%$ to $20 \%[162,164$, 165]. Carbapenems are the preferred treatment of choice for serious infections due to ESBL-producing Klebsiella pneumoniae and E. coli.

Pseudomonas aeruginosa has many resistance mechanisms and therefore demonstrates lack of susceptibility to a variety of antibiotics in liver transplant recipients. MDR Pseudomonas was the causative pathogen for healthcareassociated pneumonia (HCAP) in $18 \%$ of LT recipients [166]. Bloodstream infections due to $P$. aeruginosa have occurred in up to $10 \%$ of LT recipients, and $50 \%$ of these isolates are multidrug resistant. Treatment of MDR Pseudomonas aeruginosa can be quite challenging in LT; clinicians may often need to resort to nephrotoxic agents such as the aminoglycosides and the polymyxins. The role of cephalosporin ß-lactamase combinations such as ceftolozane/tazobactam and ceftazidime/avibactam may offer additional options for MDR Pseudomonas; however, data in the solid organ transplant patient population for these newer antimicrobial drugs is lacking.

Infections due to carbapenem-resistant Acinetobacter baumannii have been reported with increasing frequency in LT recipients, and appear to predominantly occur in the setting of HCAP and bloodstream infections. Bloodstream infections due to Acinetobacter baumannii have been reported to be as high as $24 \%$ in LT recipients, with over $50 \%$ of these isolates being caused by carbapenem-resistant A. baumannii [167-169]. Acinetobacter infections in LT recipients can carry a poor prognosis with inhospital mortality rates that may exceed $50 \%[168,170,171]$. As with MDR Pseudomonas, treatment options are limited and include the polymyxins, such as colistin, and addition of aminoglycosides. Other options include minocycline, tigecycline, and ampicillin/sulbactam, as the $\beta$-lactamase inhibitor, sulbactam, has intrinsic activity against $A$. baumannii. 
Recently, infections due to carbapenem-resistant Enterobacteriaceae (CRE) have been identified in solid organ transplant centers. Although there are multiple patterns of carbapenem resistance, the majority of cases in the USA are due to type A carbapenemases - specifically carbapenemresistant Klebsiella pneumoniae (CRKP). Rates of CRKP have been reported between $6.6 \%$ and $12.9 \%$ in patients undergoing liver transplantation, although 1 transplant center has noted a rate as high as $23 \%$ [172-174]. In general, the most common sites of infections due to CRKP in LT recipients include surgical site, organ space, HAP, and UTI [172]. Mortality associated with CRKP infections in LT recipients ranges from $18 \%$ to as high as $80 \%$ [172, 174-176]. Another cohort of solid organ transplant patients found that infection with CRKP was independently associated with higher mortality (HR 5.562 [CI 95\% 1.186-26.088]) [177]. In addition to traditional risk factors associated with CRKP infections in other patient populations, pre-transplant colonization with CRKP can place such patients at an increased risk for CRKP infections following transplantation $[175,176]$. Despite this risk, there are currently no absolute contraindications to exclude donors that are colonized with CRKP. Treatment of LT recipients who developed CRE or CRKP infections is quite complicated, often requires combination antimicrobial therapy, and can also be associated with drug toxicity. Agents that have been used include the polymyxins such as colistin, aminoglycosides, and tigecycline along with or without a carbapenem agent. Although fosfomycin can be considered for treatment of uncomplicated UTIs due to CRE, the intravenous formulation is not available in the USA. One case report of complicated extensively drug-resistant (XDR) Klebsiella pneumoniae bacteremia during early post-transplant period necessitated addition of IV fosfomycin as an investigational drug to a multidrug regimen [178]. The role of the newer $\beta$-lactamase agents such as ceftazidime/avibactam and meropenem/vaborbactam is yet to be defined for the management of serious infections due to CRE and CRKP.

Infection and colonization by vancomycin-resistant Enterococcus (VRE) have been well characterized in preliver transplant candidates and in patients following liver transplantation. In the USA, the estimated rates of VRE colonization, prior to and after transplantation, were $16 \%$ and $22 \%$, respectively [179]. There is wide variation in the prevalence of VRE colonization in LT candidates and liver transplant recipients, depending upon the transplant center and the patient population studied, and ranges from $0 \%$ to $44 \%$ [180-182]. In a prospective surveillance study, pretransplant VRE colonization was found to be associated with an increased risk for VRE infections after transplantation and associated with higher morbidity as measured by ICU stay and length of hospitalization; however, VRE colonization did not result in increased mortality. On the other hand, the acquisition of VRE colonization post-LT was associated with increased morbidity and mortality compared with LT recipients with no evidence of VRE colonization [183]. The risk factors that are associated with VRE infection include prior antibiotic exposure, prolonged hospitalization, interventional procedures or complications of the biliary tract, and surgical re-exploration [161]. Common sites of VRE infection in the LT recipient include intra-abdominal, organ space, hepatobiliary, surgical site, bloodstream, and the urinary tract. Mortality due to VRE infections in LT recipients has previously been reported to be as high as $82 \%$; this was, however, at a time when limited treatment options for VRE were available [184]. The use of linezolid and daptomycin, agents that have activity against such drug-resistant bacterial strains, has improved patient outcomes, although a $37.6 \%$ mortality has been reported in solid organ transplant recipients treated with linezolid for VRE infections [185].

Infections due to MRSA have been declining in the LT population, likely because of improved infection prevention practices such as strict implementation of hand hygiene, development of bundles for central venous catheter insertion and management, perioperative surgical site antiseptic policies, the use of daily chlorhexidine washes for select group of patients, and adherence to contact isolation policies [186]. A meta-analysis noted that there was $8.5 \%$ and 9.4\% prevalence of MRSA colonization in pre- and postliver transplant recipients, respectively. Additionally, preand post-transplant colonization was associated with six- to eleven-fold higher probability for subsequent development of MRSA invasive disease [179]. A single-center study reported $23 \%$ MRSA infection in LT recipients over an 8-year period; central vascular catheters, surgical wound, intra-abdominal space, and the lung were the most common sites of infection. Furthermore, the authors noted a $21 \%$ 30-day mortality in patients with MRSA infections [187]. Vancomycin continues to be the mainstay of treatment against MRSA; daptomycin and linezolid are being used with increasing frequency in select high-risk patients. Ceftaroline, the first cephalosporin with activity against MRSA, is approved for skin and soft tissue infections, and bacterial pneumonia, and may offer an alternative treatment option for MRSA infections in the LT patient population pending further clinical data.

The growing threat of MDRO infections in LT recipients can certainly affect patient outcomes. Therefore, emphasis on infection prevention and antibiotic stewardship is vital to limit further increases in antibiotic-resistant HAIs in patients undergoing a transplantation procedure [188]. This multidisciplinary approach has been shown to decrease surgical site infection rates by $52 \%$ in solid organ transplant recipients [189].

Infection due to Clostridium difficile has become an important healthcare-acquired pathogen with high morbidity and risk of death. The combined effect of potent immunosuppression and broad-spectrum antibiotic exposure increases 
the risk of Clostridium difficile-associated colitis during the early post-LT period. In a retrospective study of 1340 solid organ transplant recipients, the cumulative incidence of $C$. difficile colitis was highest $(3 \%)$ in liver allograft recipients [190]. In a series of 467 LT recipients, incidence was $8 \%$, with the majority of cases occurring within the first month following transplantation [191]. Another single-center retrospective study observed an incidence of $14 \%$ for $C$. difficile infection over a mean follow-up time of 1.8 years after LT; $41 \%$ of these cases occurred within 1 week after transplantation. The authors reported that most patients with $C$. difficile colitis had fever, whereas white blood cell count was less than 12,000 cells per $\mu \mathrm{L}$ [192].

\section{Fungal Infections}

In addition to bacterial infections, Candida spp. are a significant pathogen in the early post-transplant period. In a prospective multicenter investigation of invasive fungal infections following solid organ transplantation, 639 such cases among nearly 17,000 patients under surveillance were observed during the 6-year study period. Liver allograft recipients were at a high risk (41\%) among this large cohort of SOT recipients. Fungemia was prominent (44\%) disease presentation followed by intra-abdominal infection (14\%). Candida albicans and Candida glabrata species constituted the majority of infections (46\% and 24\%, respectively) [193].

In the general population, the known risk factors for developing invasive Candida infection include extended treatment with broad-spectrum antibiotics, presence of central venous catheter, use of total parenteral nutrition, presence of severe neutropenia (ANC $<500$ cells per $\mu \mathrm{L}$ ), diabetes mellitus, renal replacement therapy, mechanical ventilation, and intensive care stay. In transplant patients, recent CMV infection, primary graft failure, early surgical re-exploration, and colonization with Candida spp. during pre- and early posttransplant period are additional risk factors of invasive candidiasis. In LT recipients specifically, choledochojejunostomy is associated with a higher risk of invasive candidiasis compared to a choledochocholedochostomy anastomosis, as the former requires opening of the bowel [194]. Also, prophylaxis for spontaneous bacterial peritonitis with fluoroquinolones has been found to be an independent risk factor for invasive candidiasis in patients undergoing LT [195].

Diagnosis of invasive Candida infection is definitive when cultured from a sterile site; however, routine blood cultures lack optimal sensitivity, and often diagnosis can be missed or delayed. Detection of $\beta$-D-glucan, a polysaccharide component of fungal cell walls, can be a useful adjunctive test. However, in a multicenter analysis among LT recipients, $\beta$-D-glucan was not a reliable test for the diagnosis of invasive fungal disease [196]. Treatment for Candida spp. infection in liver transplant recipients is similar to the general population [197]. Source control, including remov- ing central venous catheters when determined as the source of fungemia, remains important for successful clearance of fungemia.

Given the high frequency of invasive Candida infection in LT population, antifungal prophylaxis after transplant surgery may be considered among patients at increased risk for fungal disease. A randomized, double-blind, placebo-controlled trial of fluconazole prophylaxis vs. no antifungal prophylaxis after LT demonstrated a decreased rate of fungal colonization and both superficial and invasive fungal infection, although no difference in mortality was found [198]. Current guidelines recommend stratification based on specific risk factors. Patients are deemed high risk for invasive candidiasis, if they have $\geq 2$ of the following conditions: prolonged or repeat operation, retransplantation, renal failure, $\geq 40$ units of cellular blood products, choledochojejunostomy, or Candida spp. colonization in the perioperative period [188]. In one series including 30 pre-LT candidates, $81 \%$ were found to have Candida carriage in gastrointestinal tract [199]. High-risk patients are recommended to be treated with an antifungal medication. Fluconazole $400 \mathrm{mg}$ daily is sufficient for most Candida spp. coverage, though, notably, prevalence of nonalbicans Candida spp. have increased and such infections are associated with higher mortality in the immunosuppressed host [194]. Duration of antifungal prophylaxis may vary by transplant center; however, 4-week duration is common. Prophylaxis may be extended based on ongoing risk factors. Recipients at low risk for invasive fungal infection may be observed without antifungal prophylaxis [200].

While Candida spp. constitute the majority of fungal infections during early post-LT period, Aspergillus spp. infections are rare, albeit a significant, pathogen. In case series and retrospective reviews, the incidence of invasive aspergillosis (IA) after LT may vary from $1 \%$ to $10 \%$ [201-203]. A multicenter surveillance network of transplant patients found an $11 \%$ incidence of IA over 5 years post-LT [204]. Multiple factors have been correlated with an increased risk of IA after transplantation. Retransplantation and the need for renal replacement therapy have the highest association [205]. It is theorized that injury to the hepatic reticuloendothelial phagocytes and alteration of platelet-mediated inflammation seen in patients with hepatic dysfunction increase susceptibility to tissue-invasive Aspergillus spp. infection [206, 207]. Renal failure may directly impact granulocyte and macrophage function or may be a marker for other predisposing factors in critically ill patients. Other factors that increase risk for IA in the initial 90 days post-LT include urgent transplantation, CMV infection, prolonged intensive care unit stay, additional surgery, and multiple invasive bacterial infections [208].

Historically, IA was most common in the first 90 days post-LT and may occur within 30 days after transplant surgery in patients at particularly high risk. However, recent 
analysis has found a slight shift in the diagnosis of IA to later post-transplantation period (>90 days) [209]. This is speculated to be due to improved overall management of transplant patients, ranging from surgical techniques to improved infection prevention and surveillance protocols. In addition, CMV infection is a known risk factor for IA, and prophylaxis against CMV may have also contributed to shift the timing of IA. Recent cases also demonstrated significantly lower mortality than in the past from $>90 \%$ down to $60 \%$; however, IA associated with early retransplantation still carries a high mortality [205, 210].

Clinically, pulmonary IA is the most common manifestation followed by disseminated disease. Diagnosis can be challenging, as cultures have low sensitivity, since isolation of Aspergillus spp. in respiratory tract culture samples in most patients represent fungal colonization. Serum fungal assays such as galactomannan and $\beta$-D-glucan are increasingly utilized to help confirm or rule out IA; however, accuracy and reliability of these assays remain uncertain [195]. Positive Aspergillus antigen from bronchoalveolar fluid has been shown to have high specificity in lung transplant recipients [211]. A combination of clinical, microbiological, and radiographic findings along with hosts' susceptibility for these infections should be considered in making diagnosis of IA in patients following LT.

Treatment of IA in liver transplant recipients is similar to the general population, as outlined in the Infectious Diseases Society of America guidelines [212]. Given the high mortality and difficulty in ascertaining correct IA diagnosis in highly susceptible transplant patients, it is recommended to initiate anti-mold therapy when IA is strongly suspected, while work-up including tissue biopsy is in progress. Voriconazole is the drug of choice for primary IA; drug interactions may warrant modified dosing for certain antirejection drugs. Concomitant reduction of immunosuppression is ideal if feasible. Adjunctive surgery may be warranted in select cases. Duration is variable, but a minimum of 12 weeks is typically recommended; however, this should be extended based on clinical and radiographic treatment response, extent of the disease, and level of net immune suppression.

While universal prophylaxis for IA is not routinely recommended following solid organ transplantation, high-risk LT recipients have been shown to benefit from it [179, 200]. This targeted prophylaxis is correlated with decreased rates of IA and appears to be cost-effective. One retrospective single-center review found administering 90 days of voriconazole prophylaxis to high-risk patients post-LT had an institutional cost of $5.6 \%$ of the predicted cost for treating IA [213]. The best choice of prophylactic antifungal is not clear. Prior studies have investigated echinocandins, liposomal amphotericin B, and voriconazole [214-216]. Echinocandins are a common choice, given their ease of single daily dosing, minimal toxicity, and drug-drug interaction. The most suit- able choice for anti-mold prophylaxis may vary depending on the clinical context.

\section{Donor-Derived Infections}

Unexpected donor-derived infections are a rare occurrence with an estimated incidence of $0.2 \%$ in solid organ transplant recipients [217]. As a result of current screening and prophylaxis practices, many viruses such as HIV, CMV, EBV, HBV, and $\mathrm{HCV}$ are identified early during the procurement period of the donor, and therefore, appropriate graft acceptance, infection surveillance, and when applicable treatment can ensue in the recipient. Unexpected donor-derived infections, although uncommon, can be an important complication particularly in the early post-transplant period. In addition to routine pre-transplant screening of the donor whether living or cadaveric allografts by history and laboratory screening, the procuring surgeon should carefully inspect the organ and donor for signs of infection by thorough physical examination. Surveillance cultures are typically sent that include blood, urine, and sputum samples. Peritoneal cultures may be warranted in cases of enterotomy and ascitic contamination to guide postsurgical antibiotic coverage [218]. Active infection in the cadaveric donor is not necessarily an absolute contraindication for transplantation; targeted antibiotics often can mitigate the risk. However, decisions are made on a case-by-case basis. Liver transplant recipients are particularly vulnerable to acquire bacterial infections via the allograft harvested from a donor with bacteremia; this possibly is related to the large tissue and vascular volume of the liver compared to other solid organ transplants [219]. Antibiotic prophylaxis may need to be extended in highrisk scenarios, such as donors with infective endocarditis and meningitis [220]. If an active bacteremia is diagnosed in the donor, the liver may still be used safely, provided that the donor has received appropriate treatment for the infection for $48 \mathrm{~h}$ and that the recipient receives at least 14 days of targeted treatment against that infection following transplantation [217, 221]. Guidelines suggest that for virulent pathogens that can cause endovascular infections such as Staphylococcus aureus and Pseudomonas aeruginosa, 2-4 weeks of antibiotic therapy should be considered although prolonged course of antibiotic therapy in recipients of such allografts has not been clinically validated [222]. Isolation of bacteria at a distant tissue site, such as in sputum or urine, usually does not necessitate antibiotic therapy in recipients of such donor allografts, with the exception of liver grafts from donors with confirmed or suspected bacterial pneumonia or pyelonephritis [222]. In some infections, such as severe encephalitis of unclear etiology, allografts are typically excluded outright [223].

In addition to bacterial etiologies, reported donortransmitted infections in LT have run the gamut and have included viruses, fungi, and parasites [224]. Uncommon 
diseases may be challenging to diagnose, and donor origin among other possible exposure and potential infection transmission history should be considered for atypical posttransplant infection. Some less common viral infections that have resulted in allograft infection transmission include West Nile virus, lymphocytic choriomeningitis virus (LCMV), and rabies; these viruses have been associated with devastating neurologic morbidity and mortality in recipients of solid organ transplantation [139, 225, 226].

The most common fungal pathogens that can be transmitted from donor to recipient are Candida species, Coccidioides immitis, and Cryptococcus neoformans [222]. Candida can be derived from the organ donor, although transmission of Candida spp. to recipients occurs more typically from contamination during the organ procurement and preservation process [227-229]. Although donor transmission of any endemic mycoses is possible, donor screening for Coccidioides immitis is the only fungal serology that is routinely obtained in donors from endemic regions. Prophylaxis with an azole antifungal agent is recommended for LT recipients whose donor has a documented positive Coccidioides serology [226]. Recently, reports of infections due to Cryptococcus neoformans occurring in the early post-transplant period have raised possibility of potential donor transmission of the dimorphic fungal infection [226, 230-232]. Donor transmission of Mycobacterium tuberculosis has been well documented in the solid organ transplant population [217, 233]. Patients with active tuberculosis infection should not be considered for organ donation; however, latent tuberculous infection in a donor is not considered contraindication for organ procurement and transplantation. In this setting, treatment of latent tuberculous infection in the recipient will mitigate the risk of developing active tuberculosis after LT.

Strongyloides stercoralis and Trypanosoma cruzi, the causative agents of Chagas disease, are the most common parasites that have been associated with donor-transmitted parasitic infections. In the solid organ transplant recipient, Strongyloides can lead to accelerated intestinal infection increasing the risk for polymicrobial bacteremia and bacterial meningitis due to enteric Gram-negative bacilli (GNB) or the rare devastating Strongyloides hyperinfection syndrome, which is associated with rapidly progressive respiratory failure and death. As a result, donor screening with Strongyloides serology should be performed in donors from endemic regions such as tropics, subtropics, and Appalachia, USA; if serology is positive, recipients should be treated with ivermectin [234, 235]. Trypanosoma cruzi donorderived infection is more problematic in patients undergoing heart transplantation; donor serologic screening for $T$. cruzi should be considered for liver allograft donors from endemic regions including Mexico and Central and South America. If a donor is positive for T. cruzi serology, there is no clear contraindication for liver transplantation, as long as the LT recipient undergoes regular surveillance for parasitemia. Preemptive treatment with benznidazole is initiated for patients with a positive T. cruzi PCR assay [236, 237].

Stored pre-transplant serum from both donor and recipient can be used to test and help confirm allograft-transmitted infection. Close collaboration with the national transplant authorities, the local organ procurement organizations, and appropriate public health agencies is vital for tracking these infections and to notify all other organ recipients from such donors.

\section{Opportunistic Infections 1 to 6-12 Months After Liver Transplantation}

After the first month, surgical recovery is well underway, and nosocomial infections become less prevalent. However, iatrogenic antirejection drug-induced immunosuppression is still high, and thus opportunistic infections are considered especially common from 1 month to 6-12 months after LT. Without prophylaxis, herpesviruses such as herpes simplex virus 1 and 2, varicella zoster virus, and cytomegalovirus, and environmental fungi like Pneumocystis jirovecii are important pathogens.

Cytomegalovirus (CMV) is the most important viral infection in LT recipients and contributes to significant morbidity and mortality in this patient population. CMV can manifest from asymptomatic viremia, to a syndrome associated with fevers and pancytopenia, to invasive target organ disease. In LT recipients, the gastrointestinal tract is the most common site of CMV involvement and can present as esophagitis, gastritis, and enterocolitis but can also involve other sites such as the lungs [238]. CMV has a predilection to involve the allograft, and therefore CMV hepatitis is an extremely common manifestation in LT recipients and can sometimes be confused with allograft rejection [239]. The incidence of CMV infection in LT recipients varies depending upon the risk group studied, with rates of $18-29 \%$ overall; however, it has been reported to be as high as $65 \%$ in the high-risk donor-recipient mismatch (D+/R-) [240-243]. The use of CMV prophylaxis with ganciclovir or valganciclovir in LT recipients for at least 3 months post-transplantation has reduced the incidence in the $\mathrm{D}+/ \mathrm{R}-$ subgroup to $12-30 \%$ [241, 243-246]. Historically, in LT recipients who do not receive $\mathrm{CMV}$ prophylaxis, $\mathrm{CMV}$ infection occurs within 3-6 months from the time of liver transplantation, which correlates with the period of maximal immunosuppression. However, in the setting of CMV prophylaxis, LT recipients can experience delayed-onset (also referred to as late-onset) CMV disease - after CMV prophylaxis has been discontinued - often occurring $>6$ months from the time of transplant [247, 248]. Delayed-onset CMV infection in LT recipients is 
more likely to cause tissue-invasive disease as compared to early-onset disease [248].

As CMV is an immunomodulatory virus, it has numerous indirect effects on the liver allograft. CMV can upregulate alloreactive $\mathrm{T}$ cells, and it can precipitate allograft rejection. In LT recipients, it has been thought to be associated with the vanishing bile duct syndrome, chronic ductopenic allograft rejection, cholestasis, and ultimately allograft failure [249252]. Chronic CMV infection, in the setting of immunosuppression, may be related to atrophy of the biliary ducts and the development of allograft arteriopathy that is seen with chronic allograft failure [253]. It is also postulated that as CMV can invade the vascular endothelium, it may be responsible for hepatic artery thrombosis [254, 255]. Finally, CMV infection can further augment the immunosuppression of transplant patients and places LT recipients at increased risk for bacterial, viral, and fungal infections, as well as increased risk for EBV-associated post-transplant lymphoproliferative disorder (PTLD) [256-258].

Risk factors for CMV infection include CMV D+/Rmismatch, lymphocyte-depleting agents such as thymoglobulin and alemtuzumab, high-dose mycophenolate mofetil, genetic polymorphisms in the toll-like receptor 2 gene, allograft rejection, and retransplantation [259, 260]. Initial infection is significantly more likely to cause symptomatic illness than reactivation [261]. There are two accepted strategies for prevention. Universal prophylaxis with valganciclovir is one approach and has the added benefit of prophylaxis against other herpesviruses. A duration of 3-6 months of prophylaxis is typical but may be extended based on clinical factors [262]. Alternatively, a preemptive approach utilizes weekly monitoring for CMV antigenemia or PCR, with prompt initiation of treatment for early replication; this strategy may be preferable for avoiding drug toxicity. There is no definitive recommendation of one approach over the other, although universal prophylaxis is preferred in highrisk transplantations - specifically CMV D+/R - allograft mismatches. A meta-analysis comparing the two in the LT population found no difference in the incidence of CMV disease. Using indirect comparison, there was also no difference in acute cellular rejection or mortality between the two groups, but a decreased incidence of graft loss with universal prophylaxis was found [263]. Treatment of CMV infection with either oral valganciclovir or intravenous ganciclovir in LT recipients is similar to other SOT recipients. Resistance to ganciclovir is rare, but more likely in patients with past prolonged use of ganciclovir [264].

Pneumocystis jirovecii is ubiquitous in the environment but transforms to a common respiratory pathogen for the immunosuppressed. Without prophylaxis, the incidence of infection in LT recipients has been found to vary from $1 \%$ to $11 \%$ [265]. Clinical presentation in SOT recipients is similar to individuals with human immunodeficiency virus (HIV), though the acuity of symptoms is typically thought to be more severe in non-HIV patients. Treatment approach is also similar to HIV patients, with trimethoprim-sulfamethoxazole (TMP/SMX) being the drug of choice and adjunctive steroids a consideration based on hypoxemia [266]. Prophylaxis against Pneumocystis is routinely recommended for the first 6-12 months in all SOT patients and may be extended as needed based on ongoing risk [267]. TMP/SMX is the first choice for prophylaxis and has the added benefit of helping to prevent other opportunistic pathogens, such as Toxoplasma gondii, Listeria monocytogenes, Nocardia spp., Isospora, Cyclospora, and some bacterial agents.

With prophylaxis for the most prevalent infections being standard, their incidences have overall been reduced, but can still occur, typically after the prophylactic course has ended. Endemic fungi and Mycobacterial spp. including $M$. tuberculosis are also not uncommon pathogens during this post-transplant period. During the time of maximal immunosuppression (1-6 months after transplantation), reactivation of endemic mycoses can occur but oftentimes present later after transplantation and may be due to exogenous infection. Cryptococcus neoformans is the third most common fungal infection in solid organ transplant recipients, with an incidence that ranges from $0.2 \%$ to $5 \%$ [268, 269]. In general, cryptococcosis is a late infectious complication, occurring a median of 16-21 months after transplantation, although in liver and lung transplant recipients, it can present earlier - within 12 months post-transplantation [269-271]. The most common sites of infection are the lungs and the central nervous system, although cutaneous, liver, kidney, and osteoarticular involvement can also occur. Disseminated and extrapulmonary cryptococcosis has been reported in 50-75\% of solid organ transplant recipients [271-273]. Liver transplant recipients have a sixfold increase risk for disseminated disease as compared to other types of transplant recipients [269]. The diagnosis and management of cryptococcosis in solid organ transplant recipients have been extrapolated from other patient populations (such as HIV) and retrospective/ observational experience and are available as practice guidelines from the Infectious Diseases Society of America and the AST Infectious Diseases Community of Practice. Initial treatment with a lipid preparation of amphotericin B and flucytosine, followed by fluconazole maintenance treatment, is recommended [269, 274].

The true incidence of endemic mycoses in the solid organ transplant population is not well defined, but infections due to Histoplasma capsulatum, Coccidioides immitis/posadasii, and Blastomyces dermatitidis have been well recognized. All of these infections can occur as a result of reactivation or as an exogenous new infection. Histoplasma capsulatum is a soil-based pathogen which has been well recognized to be endemic in the Ohio-Mississippi River Valley region of the USA. Histoplasmosis is a relatively uncommon infection in 
solid organ transplant recipients, with an incidence of $<1 \%$, even in endemic regions. Histoplasmosis usually presents within the first 2 years after transplant, but there can be wide variability regarding the time of presentation [275-278]. In solid organ transplant recipients, it presents as progressive disseminated histoplasmosis that includes pulmonary and extrapulmonary manifestations such as hepatosplenomegaly, pancytopenia, and gastrointestinal and mucosal involvement. Coccidioidomycosis is endemic in the southwestern states including Southern California, as well as Mexico, and Central America. Most cases occur within the first year of transplant, with a reported incidence of 1.4-6.9\% in endemic regions [279]. Due to depressed cellular immunity in solid organ transplant recipients, severe pneumonia, as well as dissemination to skin, bones, joints, meninges, and organ allograft can occur [280-283]. Patients with a prior history of coccidioidomycosis or a positive Coccidioides serology prior to transplantation should receive fluconazole prophylaxis after transplantation [280]. The endemic regions for blastomycosis include the Midwest, south central and southeastern regions of the USA, and provinces of Canada along the waterways. Blastomycosis is rare in solid organ transplant recipients, and an incidence of only $0.14 \%$ was noted over a 16-year observational period [284]. The treatment for the endemic mycoses includes a lipid preparation of amphotericin B and the azoles, and recommendations and guidelines for the diagnosis and management have been established [280].

Overall, a range of opportunistic infections may occur during this time of high immunosuppression post-LT, warranting a broad differential for patients presenting with infectious symptoms [285].

\section{Community-Acquired/Late Infections}

Beyond 6-12 months, graft function has ideally stabilized, and immunosuppressive medications can slowly be minimized. This decreases the net state of immunosuppression and consequently the risk of opportunistic infections; however, there is always some ongoing risk. Infections during this phase are typically community-acquired. A 5-year study at a large transplant center found a prevalence of 183 hospitalizations for infectious complications post-LT, with $145(79 \%)$ occurring in the post-6-month period. The same study found respiratory infections to be the most frequent etiology overall for solid organ recipients, accounting for $26.9 \%$ of late infections. The next most common etiologies were sepsis/bacteremia (13.1\%), liver/biliary tract (12.4\%), genitourinary $(12.2 \%), \mathrm{CMV}(7.5 \%)$, and fever of unknown origin $(8 \%)$ [286]. Community-acquired infections in posttransplant patients may also present with more severe clinical manifestations [287]. Less common pathogens, such as fungi, parasites, and mycobacteria, are also seen in the post-6-month period, sometimes related to diminished vigilance for environmental exposure prevention over time. Infections due to Nocardia spp. are relatively uncommon, with an overall incidence of $0.7-3.5 \%$ in solid organ transplant recipients and only a reported incidence of $0.1 \%$ in LT recipients [288-290]. The median time to the onset of Nocardia infection in a European cohort was 17.5 months after transplantation, and infection was associated with corticosteroid use, tacrolimus use, and elevated calcineurin trough levels within the preceding month; interestingly, the use of TMP/SMX was not protective [291]. Recently there has been an emergence of non-Aspergillus mold infections in the solid organ transplant patient population. The occurrence of these mold infections can have a bimodal distribution, and in one study, $37.8 \%$ occurred within 6 months of diagnosis, and $33 \%$ occurred $>2$ years from the time of transplantation. In that same study, the median time to development of an invasive mold infection in the LT recipients was 81 days [292]. The most common nonAspergillus molds were the Mucorales, Fusarium spp., and Scedosporium spp. The most common sites of infection for these molds were the lungs, sinuses, skin, and dissemination to the central nervous system. The dematiaceous molds that include Exophiala, Alternaria, Dactylaria, Curvularia, Cladophialophora, Verruconis gallopava, and others have been reported in the solid organ transplant populations as case reports and small case series [293]. This group of molds most often manifests as skin and soft tissue infections but can disseminate to the central nervous system. In one case series, the median time to onset was 22 months after transplantation, and the cutaneous presentation was associated with a good outcome [294].

Viral infections associated with the presence of chronic immunosuppression can also occur and include late-onset CMV infection, EBV-associated PTLD, JC virus infection associated with progressive multifocal leukoencephalopathy (PML), and HHV-8 infection associated with Kaposi's sarcoma. Liver transplant recipients have an intermediate risk for the development of EBV-associated PTLD as compared to other solid organ transplant recipients. One center reported an incidence of PTLD in $6.3 \%$ of their pediatric LT recipients and an incidence of $1.2 \%$ in the adult LT recipients [295]. The cumulative incidence of PTLD in LT recipients has been estimated to be 1-2\% over 5 years [296]. The highest risk factors for EBV-associated PTLD is due to primary infection as a result of an EBV D+/R - mismatch, in addition to a higher level of immunosuppression [297]. While the majority of cases occur within 1 year of transplantation, a $0.25 \%$ incidence has been noted in LT recipients at 1 year. Guidelines and recommendations have been established for the monitoring, diagnosis, and management of EBVassociated PTLD [298]. 
Infection with Mycobacterium tuberculosis (MTb) can occur during the period of maximal immunosuppression and well beyond that time period. Treatment of active MTb in all solid organ recipients poses a challenge for drug dosing, due to the strong interaction between rifampin (and other rifamycins) and calcineurin inhibitors or rapamycin. For LT recipients in particular, the likelihood of drug-induced hepatotoxicity is increased, warranting careful medication management [298]. Substitution of one or more first-line drugs may be needed, based on the liver function at baseline and during the course of treatment; frequent lab monitoring and consultation with a MTb expert is advised [143]. Directly observed therapy is generally preferred for transplant recipients and is vital when alternative regimens are employed. Some experts recommend a minimum 9-month treatment course for all solid organ transplant patients, due to a concern for increased mortality with shorter courses [299].

Individuals with chronic allograft dysfunction requiring higher maintenance immunosuppression should be considered at ongoing high risk for opportunistic disease and thus continued on appropriate prophylaxis as necessary.

\section{Prophylaxis/Prevention of Infections}

As detailed above, there are standard screening recommendations and protocols for prophylaxis against the most common opportunistic pathogens, particularly P. jirovecii, $C M V$, and Candida spp. Vaccination is another crucial prevention strategy. Despite the clear preventive benefits, vaccination rates have been suboptimal in LT recipients [300]. Due to chronic immune dysfunction, patients with advanced liver disease may have diminished antibody response to vaccination [301]. Consequently, it is advisable to administer vaccines as early as indicated [302]. Live vaccines are generally contraindicated post-transplant.

National guidelines for perioperative antibiotics recommend piperacillin-tazobactam or cefotaxime plus ampicillin as standard prophylaxis in liver transplantation; however, individual centers may vary their protocol [303]. A 4-year single-center review found that $53 \%$ of surgical site infections were caused by multidrug-resistant bacteria, emphasizing the need for a tailored prophylactic approach, based on patients' histories and local antibiotic resistance patterns [157].

\section{Relapse of HBV and HCV Post-liver Transplantation}

HBV reinfection rates of the allograft were previously reported to range between $80 \%$ and $100 \%$ in the 1980 s. Based on 2-year graft survival of only $50 \%$, many centers discontinued offering OLT in this population for a time [304]. Additionally, in the absence of antiviral therapy, some patients have developed fibrosing cholestatic hepatitis, a rapidly progressive and often fatal condition [305]. However, the introduction of HBIG and antiviral medications in the late 1980s has significantly improved post-transplant survival in such patients.

There are several risk factors for HBV reinfection after liver transplant. Patients at higher risk include those with positive hepatitis B e antigen ( $\mathrm{HBeAg}$ ), negative $\mathrm{HBeAg}$ but high HBV DNA level, or history of pre-transplant antiviral drug resistance. Patients at lower risk include those with cirrhosis and low HBV DNA level with or without antiviral medication, coinfection with hepatitis delta virus (HDV), or fulminant HBV infection [306].

Subclinical HBV reactivation has been reported in $\mathrm{HBsAg}$-negative and $\mathrm{HBcAb}$-positive recipients who have received livers from $\mathrm{HBs} A g$-negative and $\mathrm{HBcAb}$-negative recipients. However, this low-grade viral replication has not been associated with the development of positive HBsAg or active viral hepatitis. Therefore, antiviral therapy is not indicated in this scenario [307].

$\mathrm{HCV}$ infection recurs in the allograft in greater than $95 \%$ of $\mathrm{HCV}+$ liver allograft recipients [308]. Advanced donor age and high-intensity immunosuppression such as the use of bolus steroids or thymoglobulin can influence the severity of HCV recurrence after OLT [309, 310]. Although the course of HCV recurrence may be variable after liver transplant; up to $20 \%$ of patients develop allograft cirrhosis within 5 years of transplantation [311]. HCV+ recipients have demonstrated lower patient and graft survival when compared to patients transplanted for other indications. HIV coinfection has also correlated with diminished post-transplant survival. Fibrosing cholestatic hepatitis is a rapidly progressive condition that develops in 5-10\% of $\mathrm{HCV}+$ liver transplant recipients, at times within the first year after transplant, and often leads to diminished survival [312]. Differentiating recurrent $\mathrm{HCV}$ from ACR can be difficult due to overlapping histologic features, but certain findings such as lobular or interface hepatitis and lymphoid follicles may be more suggestive of HCV infection.

\section{HBV Prophylaxis}

All patients with $\mathrm{HBV}$ infection prior to transplantation should be continued on antiviral therapy after undergoing OLT. Additionally, patients without HBV infection who receive livers from isolated $\mathrm{HBcAb}+$ donors should also be started on antiviral therapy. Most often either entecavir or tenofovir are utilized, and the choice of a specific agent is made based on prior treatment history and side effect profiles of individual antiviral drug. 
The role of HBIG in the current era remains unclear. Prior to the discovery of potent antiviral agents, HBIG was often used long-term as standard prophylaxis with reduction in the risk for HBV recurrence [313]. However, highdose intravenous HBIG is expensive and may not provide additional benefit compared to the use of oral antiviral agents alone, especially in low-risk patients. In high-risk patients, HBIG may be discontinued after 1 year following transplantation.

\section{HCV Treatment Post-liver Transplantation}

Ideally, most patients with HCV should be treated with antiviral therapy prior to transplant. However, pre-transplant antiviral therapy may be difficult to tolerate and less effective in patients with decompensated cirrhosis. Specifically, the use of protease inhibitors for HCV is generally not recommended in patients with decompensated cirrhosis.

Currently, there are several options for treatment of genotype $1 \mathrm{HCV}$ in post-OLT patients. Ledipasvir/sofosbuvir combination therapy after OLT with weight-based ribavirin for 24 weeks has been associated with a 96\% SVR12 rate in patients with Metavir fibrosis stage F0 to F3 or those with compensated cirrhosis. In patients with decompensated cirrhosis, SVR rates range between $60 \%$ and $88 \%$ depending on degree of hepatic impairment [314]. Simeprevir and sofosbuvir with or without ribavirin for 12-24 weeks has been associated with SVR12 rates greater than $80 \%$ in both cirrhotic and non-cirrhotic patients $[315,316]$. Daclatasvir in combination with sofosbuvir with or without ribavirin for 24 weeks after OLT demonstrated a 91\% SVR12 rate in patients with severe recurrent HCV infection. The SVR12 rate was notably lower when daclatasvir was administered with simeprevir with or without ribavirin for 24 weeks [317]. The fixed-dose PrOD combination (paritaprevir, ritonavir, ombitasvir, dasabuvir) with ribavirin for 24 weeks resulted in a 96\% SVR24 rate in patients with mild fibrosis, in whom the treatment commenced after transplantation [318]. CNI troughs must be monitored carefully with the use of simeprevir or paritaprevir. Newer regimens including elbasvir/grazoprevir and sofosbuvir/velpatasvir have yet to be studied in liver transplant population with HCV infection.

There are limited data for non-genotype $1 \mathrm{HCV}$ infection in patients undergoing OLT. Daclatasvir in conjunction with sofosbuvir with or without ribavirin can be used in patients with genotype 2 or $3 \mathrm{HCV}$ infection. Patients with genotype $4 \mathrm{HCV}$ can be managed with either ledipasvir/sofosbuvir given with or without ribavirin or daclatasvir and sofosbuvir along with or without ribavirin. Sofosbuvir/velpatasvir has pangenotypic coverage and may be another option, although data in OLT recipients have yet to be reported.

\section{Other Hepatitis Virus Infections After Transplantation}

\section{Hepatitis A Infection After Liver Transplant}

Hepatitis A virus (HAV) is contracted through a fecal-oral route. Although most patients with HAV experience a selflimited course, acute liver failure may develop in less than $1 \%$ of infected individuals. Patients over 50 years of age and those with other chronic liver diseases are at higher risk to develop fulminant liver failure [319]. Accordingly, it is generally recommended that patients with chronic liver disease, including cirrhosis, should be vaccinated in the pretransplant setting. Despite vaccination, a subset of anti-HAV $\mathrm{IgG}+$ patients may lose anti-HAV IgG antibodies within the first 2 years after liver transplantation [320]. Whether loss of the anti-HAV IgG antibodies correlates with true loss of immunity and need for booster immunization(s) remains unclear. HAV vaccine is safe to administer in the post-transplant setting, although serologic response is often lower than seen in patients vaccinated prior to undergoing allograft transplantation [321]. Assuring receipt of two vaccine doses and deferring vaccination to a later time when the patient is on a lower level of immunosuppression may improve serologic response in patients requiring further vaccine doses after transplantation [322, 323]. The specific implications of HAV infection in patients undergoing liver transplantation are not well described. However, as this patient population tends to be older, and may develop chronic liver disease following OLT, the risk for severe HAV infection may be greater. Additionally, chronic drug-induced immunosuppression may promote HAV replication by subverting hosts' immune surveillance.

\section{Hepatitis E Infection After Liver Transplant}

Infection from the hepatitis $\mathrm{E}$ virus (HEV) was previously thought to be an uncommon disease in Western countries. Past reports had described a self-limited illness, which is noted more often in developing regions in Asia and SubSaharan Africa. However, HEV is being increasingly diagnosed in Europe and the USA, particularly over the past decade. Complications such as fulminant liver failure or severe hepatic decompensation have been described in a small minority of patients, particularly those who are pregnant or have chronic liver disease [324-326].

Traditionally, HEV transmission has been attributed to consumption of contaminated water and/or food, including pork products or venison. Due to its perceived rarity, most Western countries have not routinely tested blood or organ donors for HEV. However, a case of HEV transmission during blood transfusion was described in a patient following 
liver transplantation [327]. Additionally, there have been reports of potential cases of liver allograft-transmitted HEV infection with subsequent development of chronic hepatitis and cirrhosis of the transplanted allograft [328, 329].

In most immunocompetent individuals, HEV tends to cause an acute infection that resolves spontaneously. In contrast, chronic HEV infection defined as persistence of viral infection for greater than 3 months has been described in greater than $60 \%$ of solid organ transplant recipients following a primary HEV infection during the post-transplant period [330]. Among solid organ transplant (SOT), recipients of liver allografts are considered at highest risk for chronic HEV infection. Most infections are due to HEV genotype 3. In such patients, persistent HEV infection after transplantation may increase the risk for rapidly progressive hepatic fibrosis which may ultimately result in graft failure [331]. Extrahepatic manifestations of HEV infection such as cryoglobulinemia have also been reported in patients, including those who have undergone solid organ transplantation [332].

Studies from France have shown that incidence of HEV in patients undergoing OLT ranges between 2.8 and 4.8 per 100 person years $[333,334]$. Due to the variable sensitivity of HEV-IgM assays and delayed IgG response after transplantation, HEV RNA may be a more useful test for the diagnosis of chronic HEV infection in such patients [335]. Furthermore, it is important to note that SOT recipients may still contract new HEV infection despite the presence of pretransplant anti-HEV seropositivity [126].

Diagnosis of new HEV infection after transplantation should prompt a reduction in antirejection drug-related immune suppression; nearly a third of transplant recipients may resolve HEV chronic infection with this measure alone [123]. Specific immunosuppressive medications may correlate with risk of $\mathrm{HEV}$ persistence in transplant patients. In vitro studies have demonstrated increased HEV replication in the setting of tacrolimus, cyclosporine, and mTOR inhibitors [336, 337]. However, in one clinical study, liver or kidney transplant recipients who developed chronic HEV infection were more likely to have been on tacrolimus rather than cyclosporine [123]. In contrast, in vitro studies have demonstrated that mycophenolate mofetil reduces HEV replication, and this finding has been correlated in a clinical study where heart transplant patients taking mycophenolate mofetil were more likely to clear post-transplant HEV infection [338]. Corticosteroid use does not appear to affect HEV replication [129].

Patients with persistent HEV infection even after reduction of immunosuppression are candidates for antiviral therapy. In solid organ transplant recipients, ribavirin at a dose of $8 \mathrm{mg} / \mathrm{kg}$ given for at least 3 months can lead to sustained virologic response in the majority of patients [339]. Dose adjustments may be necessary based on renal function and during the course of therapy if patients develop medication-induced anemia. Smaller studies have demonstrated that PEG-interferon alpha over a 3-12 month period may lead to viral clearance, although treatment is associated with significant adverse effects and may precipitate graft rejection [340, 341].

Acknowledgments The authors express their deepest appreciation to Jonathan Kumar for his assistance with manuscript preparation.

\section{References}

1. Kamath PS, Wiesner RH, Malinchoc M, et al. A model to predict survival in patients with end-stage liver disease. Hepatology. 2001;33(2):464-70.

2. Merion RM, Schaubel DE, Dykstra DM, et al. The survival benefit of liver transplantation. Am J Transplant. 2005;5(2):307-13.

3. Angeli $\mathrm{P}$, Wong $\mathrm{F}$, Watson $\mathrm{H}$, et al. Hyponatremia in cirrhosis: results of a patient population survey. Hepatology. 2006;44(6): $1535-42$.

4. Kim WR, Biggins SW, Kremers WK, et al. Hyponatremia and mortality among patients on the liver-transplant waiting list. N Engl J Med. 2008;359(10):1018-26.

5. Ostapowicz G, Fontana RJ, Schiodt FV, et al. Results of a prospective study of acute liver failure at 17 tertiary care centers in the United States. Ann Intern Med. 2002;137(12):947-54.

6. Larson AM, Polson J, Fontana RJ, et al. Acetaminophen-induced acute liver failure: results of a United States multicenter, prospective study. Hepatology. 2005;42(6):1364-72.

7. Mazzaferro V, Regalia E, Doci R, et al. Liver transplantation for the treatment of small hepatocellular carcinomas in patients with cirrhosis. N Engl J Med. 1996;334(11):693-9.

8. Yao FY. Liver transplantation for hepatocellular carcinoma: beyond the Milan criteria. Am J Transplant. 2008;8(10):1982-9.

9. Darwish Murad S, Kim WR, Harnois DM, et al. Efficacy of neoadjuvant chemoradiation, followed by liver transplantation for perihilar cholangiocarcinoma at 12 US centers. Gastroenterology. 2012;143(1):88-98.

10. Mathurin P, Moreno C, Samuel D, et al. Early liver transplantation for severe alcoholic hepatitis. N Engl J Med. 2011;365(19):1790-800.

11. Terrault NA, Roland ME, Schiano T, et al. Outcomes of liver transplant recipients with hepatitis $\mathrm{C}$ and human immunodeficiency virus coinfection. Liver Transpl. 2012;18(6):716-26.

12. Abt PL, Desai NM, Crawford MD, et al. Survival following liver transplantation from controlled non-heart-beating donors: an increased incidence of biliary complications. Transplantation. 2004;239:87-92.

13. Manzarbeitia CY, Ortiz JA, Jeon H, et al. Long-term outcome of controlled non-heart-beating donor liver transplantation. Transplantation. 2004;78:211-5.

14. Mateo R, Cho Y, Singh G, et al. Risk factors for graft survival after liver transplantation from donation after cardiac death donors: an analysis of OPTN/UNOS data. Am J Transplant. 2006;6:791-6.

15. Lee KW, Simpkins CE, Montgomery RA, et al. Factors affecting graft survival after liver transplantation from donation after cardiac death donors. Transplantation. 2006;82:1683-8.

16. Freise CE, Gillespie BW, Koffron AJ, et al. Recipient morbidity after living and deceased donor liver transplantation: findings from the A2ALL retrospective cohort study. Am J Transplant. 2008;8(12):2569-79.

17. Olthoff KM, Smith AR, Abecassis M, et al. Defining long-term outcomes with living donor liver transplantation in North America. Ann Surg. 2015;262(3):465-75. 
18. Wan P, Yu X, Xia Q. Operative outcomes of adult living donor liver transplantation and deceased donor liver transplantation: a systematic review and meta-analysis. Liver Transpl. 2014;20(4):425-36.

19. A definition of irreversible coma Report of the Ad Hoc Committee of the Harvard Medical School to Examine the Definition of Brain Death. JAMA. 1968;2015:337-40.

20. Guidelines for the determination of death Report of the medical consultants on the diagnosis of death to the President's Commission for the Study of Ethical Problems in Medicine and Biomedical and Behavioral Research. JAMA. 1981;246:2184-6.

21. Guideline three: minimum technical standards for EEG recording in suspected cerebral death. J Clinical Neurophysiol. 1994;11:10-3.

22. Roper AH, Kehne SM, Weschler L. Transcranial Doppler in brain death. Neurology. 1987;37:1733-5.

23. Wall WJ, Mimeault R, Grant DR, et al. The use of older donor livers for hepatic transplantation. Transplantation. 1990;49:377-81.

24. Cescon M, Grazi GL, Cucchetti A. Improving the outcome of liver transplantation with very old donors with updated selection and management criteria. Liver Transpl. 2000;14:672-9.

25. Nardo B, Masetti M, Urbani L, et al. Liver transplantation from donors aged 80 years and over: pushing the limit. Am J Transplant. 2004;4:1139-47.

26. Veretemati M, Sabatella G, Minola E. Morphometric analysis of primary graft non-function in liver transplantation. Histopathology. 2005;46:451-9.

27. Imber CJ, St Peter SD, Handa A, et al. Hepatic steatosis and its relationship to transplantation. Liver Transpl. 2002;8:415-23.

28. Feng S, Goodrich NP, Bragg-Gresham JL, et al. Characteristics associated with liver graft failure: the concept of a donor risk index. Am J Transplant. 2006;6:783-90.

29. Ellingson K, Seem D, Nowicki M, et al. Estimated risk of human immunodeficiency virus and hepatitis $\mathrm{C}$ virus infection among potential organ donors from 17 organ procurement organizations in the United States. Am J Transplant. 2011;11:1201-8.

30. Seem DL, Lee I, Umscheid CA, et al. PHS guideline for reducing human immunodeficiency virus, hepatitis B virus, and hepatitis $\mathrm{C}$ virus transmission through organ transplantation. Public Health Rep. 2013;128:247-343.

31. Munoz SJ. Use of hepatitis B core antibody-positive donors for liver transplantation. Liver Transpl. 2002;8(10 Suppl 1):S82-7.

32. Saab S, Waterman B, Chi AC, et al. Comparison of different immunoprophylaxis regimens after liver transplantation with hepatitis B core antibody-positive donors: a systematic review. Liver Transpl. 2010;16(3):300-7.

33. Vargas HE, Laskus T, Wang LF, et al. Outcome of liver transplantation in hepatitis $\mathrm{C}$ virus-infected patients who received hepatitis $\mathrm{C}$ virus-infected grafts. Gastroenterology. 1999;117(1):149-53.

34. Duffy JP, Hong JC, Farmer DG, et al. Vascular complications of orthotopic liver transplantation: experience in more than 4,200 patients. J Am Coll Surg. 2009;208(5):896-903.

35. Welling TH, Heidt DG, Englesbe MJ, et al. Biliary complications following liver transplantation in the model for end-stage liver disease era: effect of donor, recipient, and technical factors. Liver Transpl. 2008;14(1):73-80.

36. Jay CL, Lyuksemburg V, Ladner DP, et al. Ischemic cholangiopathy after controlled donation after cardiac death liver transplantation: a meta-analysis. Ann Surg. 2011;253(2):259-64.

37. Dacha S, Barad A, Martin J, et al. Association of hepatic artery stenosis and biliary strictures in liver transplant recipients. Liver Transpl. 2011;17(7):849-54.

38. Motschman TL, Taswell HF, Brecher ME, et al. Intraoperative blood loss and patient and graft survival in orthotopic liver transplantation: their relationship to clinical and laboratory data. Mayo Clin Proc. 1989;64(3):346-55.

39. U.S. Multicenter FK506 Liver Study Group. A comparison of tacrolimus (FK506) and cyclosporine for immunosuppression in liver transplantation. The U.S. Multicenter FK506 Liver Study Group. N Engl J Med. 1994;331(17):1110-5.

40. O'Grady JG, Burroughs A, Hardy P, et al. Tacrolimus versus microemulsified ciclosporin in liver transplantation: the TMC randomised controlled trial. Lancet. 2002;360(9340):1119-25.

41. McAlister VC, Haddad E, Renouf E, et al. Cyclosporin versus tacrolimus as primary immunosuppressant after liver transplantation: a meta-analysis. Am J Transplant. 2006;6(7):1578-85.

42. Asrani SK, Leise MD, West CP, et al. Use of sirolimus in liver transplant recipients with renal insufficiency: a systematic review and meta-analysis. Hepatology. 2010;52(4):1360-70.

43. Rogers CC, Johnson SR, Mandelbrot DA, et al. Timing of sirolimus conversion influences recovery of renal function in liver transplant recipients. Clin Transpl. 2009;23(6):887-96.

44. Sterneck M, Kaiser GM, Richter N, et al. Everolimus and early calcineurin inhibitor withdrawal: 3-year results from a randomized trial in liver transplantation. Am J Transplant. 2014;14(3):701-10.

45. Euvrard S, Morelon E, Rostaing L, et al. Sirolimus and secondary skin-cancer prevention in kidney transplantation. N Engl J Med. 2012;367(4):329-39.

46. Zimmerman MA, Trotter JF, Wachs M, et al. Sirolimus-based immunosuppression following liver transplantation for hepatocellular carcinoma. Liver Transpl. 2008;14(5):633-8.

47. Geissler EK, Schnitzbauer AA, Zulke C, et al. Sirolimus use in liver transplant recipients with hepatocellular carcinoma: a randomized, multicenter, open-label phase 3 trial. Transplantation. 2016;100(1):116-25.

48. Lake J, Patel D, David K, et al. The association between MMF and risk of progressive renal dysfunction and death in adult liver transplant recipients with HCV. Clin Transpl. 2009;23(1):108-15.

49. Vivarelli M, Burra P, La Barba G, et al. Influence of steroids on HCV recurrence after liver transplantation: a prospective study. J Hepatol. 2007;47(6):793-8.

50. Eckhoff DE, McGuire B, Sellers M, et al. The safety and efficacy of a two-dose daclizumab (zenapax) induction therapy in liver transplant recipients. Transplantation. 2000;69(9):1867-72.

51. Wiesner RH, Menon KV. Late hepatic allograft dysfunction. Liver Transpl. 2001;7(11 Supple 1):S60-73.

52. Thurairajah PH, Carbone M, Bridgestock $\mathrm{H}$, et al. Late acute liver allograft rejection; a study of its natural history and graft survival in the current era. Transplantation. 2013;95(7):955-9.

53. Banff schema for grading liver allograft rejection: an international consensus document. Hepatology. 1997;25(3):658-63.

54. Wiesner RH, Batts KP, Krom RA. Severe ductopenic rejection following liver transplantation: incidence, time of onset, risk factors, treatment, and outcome. Liver Transpl Surg. 1999;5(5):388-400.

55. Wong F, Bernardi M, Balk R, et al. Sepsis in cirrhosis: report on the 7th meeting of the International Ascites Club. Gut. 2005;54:718-25.

56. Fernandez J, Arroyo V. Bacterial infections in cirrhosis: a growing problem with significant implications. Clin Liver Dis. 2013;2:102-5.

57. Bunchorntavakul C, Chamroonkul N, Chavalitdhamrong D. Bacterial infections in cirrhosis: a critical review and practical guidance. World J Hepatol. 2016;8(6):307-21.

58. Robinson MW, Harmon C, O'Farrelly C. Liver immunology and its role in inflammation and homeostasis. Cell Mol Immunol. 2016;13(3):267-76.

59. Bilzer M, Roggel F, Gerbes AL. Role of Kupffer cells in host defense and liver disease. Liver Int. 2006;26:1175-86.

60. Janeway CA. The immune system evolved to discriminate infectious nonself from noninfectious self. Immunol Today. 1992;13:11-6.

61. Takeuchi O, Akira S. Pattern recognition receptors and inflammation. Cell. 2010;140:805-20.

62. Kubes P, Mehal WZ. Sterile inflammation in the liver. Gastroenterology. 2012;143:1158-72.

63. Crispe IN. Immune tolerance in liver disease. Hepatology. 2014;60:2109-17. 
64. Goel A, Gupta M, Aggarwal R. Gut microbiota and liver disease. J Gastroenterol Hepatol. 2014;29(6):1139-48.

65. Teltschik Z, Wiest R, Beisner J, et al. Intestinal bacterial translocation in rats with cirrhosis is related to compromised Paneth cell antimicrobial host defense. Hepatology. 2012;55:1154-63.

66. Steffen EK, Berg RD, Deitch EA. Comparison of translocation rates of various indigenous bacteria from the gastrointestinal tract to the mesenteric lymph node. J Infect Dis. 1988;157:1032-8.

67. Lakshmi CP, Ghoshal UC, Kumar S, et al. Frequency and factors associated with small intestinal bacterial overgrowth in patients with cirrhosis of the liver and extra hepatic portal venous obstruction. Dig Dis Sci. 2010;55:1142-8.

68. Gupta A, Dhiman RK, Kumari S, et al. Role of small intestinal bacterial overgrowth and delayed gastrointestinal transit time in cirrhotic patients with minimal hepatic encephalopathy. J Hepatol. 2010;53:849-55.

69. Liu Q, Duan ZP, Ha DK, et al. Symbiotic modulation of gut flora: effect on minimal hepatic encephalopathy in patients with cirrhosis. Hepatology. 2004;39:1441-9.

70. Assimakopoulos SF, Tsamandas AC, Tsiaoussis GI, et al. Altered intestinal tight junctions' expression in patients with liver cirrhosis: a pathogenetic mechanism of intestinal hyperpermeability. Eur J Clin Investig. 2012;42:439-46.

71. Assimakopoulos SF. Uncovering the molecular events associated with increased intestinal permeability in liver cirrhosis: the pivotal role of enterocyte tight junctions and future perspectives. J Hepatol. 2013;59:1144-6.

72. Saitoh O, Sugi K, Lojima K, et al. Increased prevalence of intestinal inflammation in patients with liver cirrhosis. World J Gastroenterol. 1999;5:391-6.

73. Lorenzo-Zuniga V, Bartoli R, Planas R, et al. Oral bile acids reduce bacterial overgrowth, bacterial translocation, and endotoxemia in cirrhotic rats. Hepatology. 2003;37:551-7.

74. Jenne CN, Kubes P. Immune surveillance by the liver. Nat Immunol. 2013;14(10):996-1006.

75. Guarner-Argente C, Sánchez E, Vidal S, et al. Toll-like receptor 4 D299G polymorphism and the incidence of infections in cirrhotic patients. Aliment Pharmacol Ther. 2010;31:1192-9.

76. Nischalke HD, Berger C, Aldenhoff K, et al. Toll-like receptor (TLR) 2 promoter and intron 2 polymorphisms are associated with increased risk for spontaneous bacterial peritonitis in liver cirrhosis. J Hepatol. 2011;55:1010-6.

77. Jalan R, Fernandez J, Wiest R, et al. Bacterial infections in cirrhosis: a position statement based on the EASL special conference 2013. J Hepatol. 2014;60:1310-24.

78. Tritto G, Bechlis Z, Stadlbauer V, et al. Evidence of neutrophil functional defect despite inflammation in stable cirrhosis. J Hepatol. 2011;55:574-81.

79. Doi H, Iyer TK, Carpenter E, et al. Dysfunctional B-cell activation in cirrhosis resulting from hepatitis $\mathrm{C}$ infection associated with disappearance of CD27-positive B-cell population. Hepatology. 2012;55:709-19.

80. Tian Z, Chen Y, Gao B. Natural killer cells in liver disease. Hepatology. 2013;57:1654-62.

81. Lario M, Munoz L, Ubeda M, et al. Defective thymopoiesis and poor peripheral homeostatic replenishment of T-helper cells cause T-cell lymphopenia in cirrhosis. J Hepatol. 2013;59:723-30.

82. Laso FJ, Madruga JI, Giron JA, et al. Decreased natural killer cytotoxic activity in chronic alcoholism is associated with alcohol liver disease but not active ethanol consumption. Hepatology. 1997;25:1096-100.

83. Wasmuth HE, Kunz D, Yagmur E, et al. Patients with acute on chronic liver failure display "sepsis like" immune paralysis. J Hepatol. 2005;42:195-201.

84. Gao B, Jeong WI, Tian Z. Liver: an organ with predominant innate immunity. Hepatology. 2008;47(2):729-36.
85. Ramadori G, Christ B. Cytokines and the hepatic acute-phase response. Semin Liver Dis. 1999;19(2):141-55.

86. Liu C, Xu Z, Gupta D, et al. Peptidoglycan recognition proteins: a novel family of four human innate immunity pattern recognition molecules. J Biol Chem. 2001;276(37):34686-94.

87. Albillos A, Lario M, Álvarez-Mon M. Cirrhosis-associated immune dysfunction: distinctive features and clinical relevance. J Hepatol. 2014;61:1385-96.

88. Runyon BA, Morrissey RL, Hoefs JC, et al. Opsonic activity of human ascitic fluid: a potentially important protective mechanism against spontaneous bacterial peritonitis. Hepatology. 1985;5(4):634-7.

89. Runyon BA. Patients with deficient ascitic fluid opsonic activity are predisposed to spontaneous bacterial peritonitis. Hepatology. 1988;8(3):632-5.

90. Rajkovic IA, Williams R. Abnormalities of neutrophil phagocytosis, intracellular killing and metabolic activity in alcoholic cirrhosis and hepatitis. Hepatology. 1986;6(2):252-62.

91. Szabo G, Saha B. Alcohol's effect on host defense. Alcohol Res. 2015;37(2):159-70.

92. Tandon P, Garcia-Tsao G. Bacterial infections, sepsis, and multiorgan failure in cirrhosis. Semin Liver Dis. 2008;28(1):26-42.

93. Bonnel AR, Bunchorntavakul C, Reddy KR. Immune dysfunction and infections in patients with cirrhosis. Clin Gastroenterol Hepatol. 2011;9:727-38.

94. Barnes PF, Arevalo C, Chan LS, Wong SF, Reynolds TB. A prospective evaluation of bacteremic patients with chronic liver disease. Hepatology. 1988;8:1099-103.

95. Fernández J, Navasa M, Gómez J, Colmenero J, Vila J, Arroyo V, Rodés J. Bacterial infections in cirrhosis: epidemiological changes with invasive procedures and norfloxacin prophylaxis. Hepatology. 2002;35:140-8.

96. Caly WR, Strauss E. A prospective study of bacterial infections in patients with cirrhosis. J Hepatol. 1993;18(3):353-8.

97. Brann OS. Infectious complications of cirrhosis. Curr Gastroenterol Rep. 2001;3:285-92.

98. Merli M, Lucidi C, Giannelli V, Giusto M, Riggio O, Falcone M, et al. Cirrhotic patients are at risk for health care-associated bacterial infections. Clin Gastroenterol Hepatol. 2010;8:979-85.

99. Bunchorntavakul C, Chavalitdhamrong D. Bacterial infections other than spontaneous bacterial peritonitis in cirrhosis. World J Hepatol. 2012;4:158-68.

100. McHutchison JG, Runyon BA. Spontaneous bacterial Peritonitis. In: Surawicz CM, Owen RL, editors. Gastrointestinal and hepatic infections. Philadelphia: WB Saunders; 1995. p. 455.

101. Bert F, Noussair L, Lambert-Zechovsky N, Valla D. Viridans group streptococci: an underestimated cause of spontaneous bacterial peritonitis in cirrhotic patients with ascites. Eur J Gastroenterol Hepatol. 2005;17:929-3.

102. Runyon BA. Introduction to the revised American Association for the Study of Liver Diseases Practice Guideline management of adult patients with ascites due to cirrhosis 2012. Hepatology. 2013;57:1651.

103. Mandell LA, Wunderink RG, Anzueto A, Bartlett JG, Campbell GD, Dean NC, et al. Infectious Diseases Society of America/ American Thoracic Society consensus guidelines on the management of community-acquired pneumonia in adults. Clin Infect Dis. 2007;44(Suppl 2):S27-72.

104. Happel KI, Nelson S. Alcohol, immunosuppression, and the lung. Proc Am Thorac Soc. 2005;2:428-32.

105. Fuxench-Lopez Z, Ramirez-Ronda CH. Pharyngeal flora in ambulatory alcoholic patients: prevalence of gram-negative bacilli. Arch Intern Med. 1978;138:1815-6.

106. Marik PE. The clinical features of severe community-acquired pneumonia presenting as septic shock. Norasept II Study Investigators. J Crit Care. 2000;15:85-90. 
107. Campillo B, Richardet JP, Kheo T, et al. Nosocomial spontaneous bacterial peritonitis and bacteremia in cirrhotic patients: impact of isolate type on prognosis and characteristics of infection. Clin Infect Dis. 2002;35:1-10.

108. Bernard B, Grangé JD, Khac EN, et al. Antibiotic prophylaxis for the prevention of bacterial infections in cirrhotic patients with gastrointestinal bleeding: a meta-analysis. Hepatology. 1999;29:1655-61.

109. Jia Y, Dwivedi A, Elhanafi S, et al. Low risk of bacteremia after endoscopic variceal therapy for esophageal varices: a systematic review and meta-analysis. Endosc Int Open. 2015;3(5): E409-17.

110. Sauerbruch T, Holl J, Ruckdeschel G, et al. Bacteremia associated with endoscopic sclerotherapy of oesophageal varices. Endoscopy. 1985;17(5):170-2.

111. Maulaz EB, de Mattos AA, Pereira-Lima J, et al. Bacteremia in cirrhotic patients submitted to endoscopic band ligation of esophageal varices. Arq Gastroenterol. 2003;40(3):166-72.

112. Cho SY, Kang CI, Kim J, et al. Association of liver cirrhosis with group B streptococcal bacteremia in non-pregnant adults. J Infect. 2013;67(6):617-9.

113. Xiol X, Castellví JM, Guardiola J, et al. Spontaneous bacterial empyema in cirrhotic patients: a prospective study. Hepatology. 1996;23:719-23.

114. Chen TA, Lo GH, Lai KH. Risk factors for spontaneous bacterial empyema in cirrhotic patients with hydrothorax. J Chin Med Assoc. 2003;66:579-86.

115. Chen $\mathrm{CH}$, Shih CM, Chou JW, et al. Outcome predictors of cirrhotic patients with spontaneous bacterial empyema. Liver Int. 2011;31:417-24.

116. Michels K, Nemeth E, Ganz T, et al. Hepcidin and host defense against infectious diseases. PLoS Pathog. 2015;11:e1004998.

117. Milic S, Mikolasevic I, Orlic L, et al. The role of iron and iron overload in chronic liver disease. Med Sci Monit. 2016;22:2144-51.

118. Arezes J, Jung G, Gabayan V, et al. Hepcidin-induced hypoferremia is a critical host defense mechanism against the siderophilic bacterium Vibrio vulnificus. Cell Host Microbe. 2015;17(1):47-57.

119. Khan FA, Fisher MA, Khakis RA. Association of hemochromatosis with infectious diseases: expanding spectrum. Int J Infect Dis. 2007;11(6):482-7.

120. Blake PA, Merson MH, Weaver RE, et al. Disease caused by a marine Vibrio. Clinical characteristics and epidemiology. N Engl J Med. 1979;300:1

121. Dechet AM, Yu PA, Koram N. Non-foodborne Vibrio infections: an important cause of morbidity and mortality in the United States, 1997-2006. Clin Infect Dis. 2008;46:970.

122. Tacket CO, Brenner F, Blake PA. Clinical features and an epidemiological study of Vibrio vulnificus infections. J Infect Dis. 1984;149:558

123. Jones MK, Oliver JD. Vibrio vulnificus: disease and pathogenesis. Infect Immun. 2009;77:1723.

124. Yoder JS, Hlavsa MC, Craun GF, et al. Surveillance for waterborne disease and outbreaks associated with recreational water use and other aquatic facility-associated health events - United States, 2005-2006. MMWR Surveill Summ. 2008;57:1.

125. Liu JW, Lee IK, Tang HJ, et al. Prognostic factors and antibiotics in Vibrio vulnificus septicemia. Arch Intern Med. 2006;166(19):2117.

126. Ko WC, Chuang YC. Aeromonas bacteremia: review of 59 episodes. Clin Infect Dis. 1995;20:1298-304.

127. Choi JP, Lee SO, Kwon HH, et al. Clinical significance of spontaneous Aeromonas bacterial peritonitis in cirrhotic patients: a matched case-control study. Clin Infect Dis. 2008;47(1):66-72.

128. Alcañiz JP, de Cuenca Morón B, Gómez Rubio M, et al. Spontaneous bacterial peritonitis due to Plesiomonas shigelloides. Am J Gastroenterol. 1995;90:1529-30.
129. Patel S, Gandhi D, Mehta V, et al. Plesiomonas shigelloides: an extremely rare cause of spontaneous bacterial peritonitis. Acta Gastroenterol Belg. 2016;79(1):52-3.

130. Centers for Disease Control and Prevention (CDC). Red blood cell transfusions contaminated with Yersinia enterocolitica - United States, 1991-1996, and initiation of a national study to detect bacteria-associated transfusion reactions. MMWR Morb Mortal Wkly Rep. 1997;46:553.

131. van Asbeck BS, Verbrugh HA, van Oost BA, et al. Listeria monocytogenes meningitis and decreased phagocytosis associated with iron overload. Br Med J (Clin Res Ed). 1982;284(6315):524.

132. Mossey RT, Sondheimer J. Listeriosis in patients with longterm hemodialysis and transfusional iron overload. Am J Med. 1985;79:397.

133. Mook P, O'Brien SJ, Gillespie IA. Concurrent conditions and human listeriosis, England, 1999-2009. Emerg Infect Dis. 2011:17:38.

134. Winston DJ, Emmanouilides C, Busuttil RW. Infections in liver transplant recipients. Clin Infect Dis. 1995;21:1077-91.

135. Fishman JA. Infection in solid-organ transplant recipients. N Engl J Med. 2007;357(25):2601-14.

136. Fishman JA. Infection in organ transplantation. Am J Transplant. 2017;17(4):856-79.

137. Domen RE, Nelson KA. Results of a survey of infectious disease testing practices by organ procurement organization in the United States. Transplantation. 1997;63(12):1790-4.

138. Delmonico FL, Snydman DR. Organ donor screening for infectious diseases: review of practice and implications for transplantation. Transplantation. 1998;65(5):603-10.

139. Fischer SA, Lu K, the AST Infectious Diseases Community of Practice. Screening of donor and recipient in solid organ transplantation. Am J Transplant. 2013;13:9-21.

140. Cohn DL, O'Brien RJ, Geiter LJ, et al. Targeted tuberculin testing and treatment of latent tuberculosis infection. American Thoracic Society. MMWR Recomm Rep. 2000;49(RR-6):1-51.

141. Mazurek GH, Jerob J, Vernon A, et al. Updated guidelines for using interferon gamma release assays to detect mycobacterium tuberculosis infection - United States, 2010. MMWR Recomm Rep. 2010;59(RR05):1-25.

142. Schirren CA, Jung MC, Zachoval R, et al. Analysis of T-cell activation pathways in patients with liver cirrhosis, impaired delayed hypersensitivity and other T-cell dependent functions. Clin Exp Immunol. 1997;108:144-50.

143. Lindemann M, Dioury Y, Beckebaum S, et al. Diagnosis of tuberculosis infection in patients awaiting liver transplantation. Hum Immunol. 2009;70:24-8.

144. Casas S, Munoz L, Moure R, et al. Comparison of the 2-step tuberculin skin test and the quantiFERON-TB gold in-tube test for the screening of tuberculosis infection before liver transplantation. Liver Transpl. 2011;17(10):1205-11.

145. Theodoropoulos N, Lanternier F, Rassiwala J, et al. Use of the QuantiFERON-TB gold interferon-gamma release assay for screening transplant candidates: a single-center retrospective study. Transpl Infect Dis. 2012;14(1):1-8.

146. Subramanian AK, Morris MI, the AST Infectious Diseases Community of Practice. Mycobacterium tuberculosis infections in solid organ transplantation. Am J Transplant. 2013;13: 68-76.

147. Pai M, Zwerling A, Menzies D. Systematic review: T-cell-based assays for the diagnosis of latent tuberculosis infection: an update. Ann Intern Med. 2008;149:177-84.

148. Jhang AW, Tran T, Bui L, et al. Safety of treatment of latent tuberculosis infection in compensated cirrhotic patients during transplant candidacy period. Transplantation. 2007;83:1557-62.

149. Singh N, Wagener MM, Gayowski T. Safety and efficacy of isoniazid chemoprophylaxis administered during liver transplant 
candidacy for the prevention of post-transplant tuberculosis Transplantation. 2002;74:892-5.

150. Centers for Disease Control and Prevention. Targeted tuberculin testing and treatment of latent tuberculosis infection. MMWR. 2000;49(No.RR-6):7.

151. Torre-Cisneros J, San-Juan R, Rosso-Fernández CM, et al. Tuberculosis prophylaxis with levofloxacin in liver transplant patients is associated with a high incidence of tenosynovitis: safety analysis of a multicenter randomized trial. Clin Infect Dis. 2015;60(11):1642-9.

152. Del Pilar Hernandez M, Martin P, Simkins J. Infectious complications after liver transplantation. Gastroenterol Hepatol. 2015;11(11):741-53.

153. Kaltenborn A, Gutcke A, Gwiasda J, et al. Biliary complications following liver transplantation: single center experience over three decades and recent risk factors. World J Hepatol. 2017;9(3):147-54.

154. Tachopoulou OA, Vogt DP, Henderson JM, et al. Hepatic abscess after liver transplantation: 1990-2000. Transplantation. 2003;72(1):79-83.

155. Santos CA, Hotchkiss RS, Chapman WC, et al. Epidemiology of bloodstream infections in a multicenter retrospective cohort of liver transplant recipients. Transplant Dir. 2016;2(3):e67.

156. Freire MP, Soares Oshiro IC, Bonazzi PR, et al. Surgical site infections in liver transplant recipients in the model for end-stage liver disease era: an analysis of the epidemiology, risk factors, and outcomes. Liver Transpl. 2013;19(9):1011-9.

157. Viehman JA, Clancy CJ, Clarke L, et al. Surgical site infections after liver transplantation: emergence of multidrug-resistant bacteria and implications for prophylaxis and treatment strategies. Transplantation. 2016;100:2107-14.

158. Weiner LM, Fridkin SK, Aponte-Torres Z, et al. Vital signs: preventing antibiotic-resistant infections in hospitals - United States, 2014. Am J Transplant. 2016;16(7):2224-30.

159. Weiner LM, Webb AK, Limbago B, et al. Antimicrobial-resistant pathogens associated with healthcare-associated infections: summary of data reported to the National Healthcare Safety Network at the Centers for Disease Control and Prevention, 2011-2014. Infect Control Hosp Epidemiol. 2016;37(11):1288-301.

160. van Duin D, van Delden C, the AST Infectious Diseases Community of Practice. Multidrug-resistant gram-negative bacteria infections in solid organ transplantation. Am J Transplant. 2013;13(suppl 4):31-41.

161. Santoro-Lopes G, de Gouvêa EF. Multidrug-resistant bacterial infections after liver transplantation: an ever-growing challenge. World J Gastroenterol. 2014;20(20):6201-10.

162. Linares L, Cervera C, Hoyo I, et al. Klebsiella pneumoniae infection in solid organ transplant recipients: epidemiology and antibiotic resistance. Transplant Proc. 2010;42(8):2941-3.

163. Bert F, Larroque B, Paugam-Burtz C, et al. Pretransplant fecal carriage of extended-spectrum $\beta$-lactamase-producing Enterobacteriaceae and infection after liver transplant, France. Emerg Infect Dis. 2012;18:908-16.

164. Rebuck JA, Olsen KM, Fey PD, et al. Characterization of an outbreak due to extended-spectrum beta-lactamase-producing Klebsiella pneumoniae in a pediatric intensive care unit transplant population. Clin Infect Dis. 2000;31:1368-72.

165. Winters HA, Parbhoo RK, Schafer JJ, et al. Extended-spectrum beta-lactamase-producing bacterial infections in adult solid organ transplant recipients. Ann Pharmacother. 2011;45:309-16.

166. Cervera C, Agustí C, Angeles Marcos M, et al. Microbiologic features and outcome of pneumonia in transplanted patients. Diagn Microbiol Infect Dis. 2006;55:47-54.

167. Shi SH, Kong HS, Xu J, et al. Multidrug resistant gram-negative bacilli as predominant bacteremic pathogens in liver transplant recipients. Transpl Infect Dis. 2009;11:405-12.
168. Liu H, Ye Q, Wan Q, et al. Predictors of mortality in solid-organ transplant recipients with infections caused by Acinetobacter baumannii. Ther Clin Risk Manag. 2015;11:1251-7.

169. Ye QF, Zhao J, Wan QQ, et al. Frequency and clinical outcomes of ESKAPE bacteremia in solid organ transplantation and the risk factors for mortality. Transpl Infect Dis. 2014;16:767-74.

170. Shields RK, Clancy CJ, Gillis LM, et al. Epidemiology, clinical characteristics and outcomes of extensively drug-resistant Acinetobacter baumannii infections among solid organ transplant recipients. PLoS One. 2012;7:e52349.

171. Kim YJ, Yoon JH, Kim SI, Hong KW, Kim JI, Choi JY, et al. High mortality associated with Acinetobacter species infection in liver transplant patients. Transplant Proc. 2011;43:2397-9.

172. Pereira MR, Scully BF, Pouch SM, et al. Risk factors and outcomes of carbapenem-resistant Klebsiella pneumoniae infections in liver transplant recipients. Liver Transpl. 2015;21:1511-9.

173. Bergamasco MD, Barros O, Barbosa M, et al. Infection with Klebsiella pneumoniae carbapenemase (KPC)-producing $K$. pneumoniae in solid organ transplantation. Transpl Infect Dis. 2012;14:198-205.

174. Kalpoe JS, Sonnenberg E, Factor SH, et al. Mortality associated with carbapenem-resistant Klebsiella pneumoniae infections in liver transplant recipients. Liver Transpl. 2012;18:468-74.

175. Giannella M, Bartoletti M, Morelli MC, et al. Risk factors for infection with carbapenem-resistant Klebsiella pneumoniae after liver transplantation: the importance of pre- and posttransplant colonization. Am J Transplant. 2015;15:1708-15.

176. Lübbert C, Becker-Rux D, Rodloff AC, et al. Colonization of liver transplant recipients with KPC-producing Klebsiella pneumoniae is associated with high infection rates and excess mortality: a case-control analysis. Infection. 2014;42:309-16.

177. Barchiesi F, Montalti R, Castelli P, et al. Carbapenem-resistant Klebsiella pneumoniae influences the outcome of early infections in liver transplant recipients. BMC Infect Dis. 2016;16(1):538.

178. Mills JP, Wilck MB, Weikert BC, et al. Successful treatment of a disseminated infection with extensively drug-resistant Klebsiella pneumoniae in a liver transplant recipient with a fosfomycinbased multidrug regimen. Transpl Infect Dis. 2016;18(5): $777-81$.

179. Ziakas PD, Pliakos EE, Zervou FN, et al. MRSA and VRE colonization in solid organ transplantation: a meta-analysis of published studies. Am J Transplant. 2014;14:1887-94.

180. de Gouvea EF, Castelo Branco R, Monteiro RC, et al. Surveillance for vancomycin-resistant enterococci colonization among patients of a liver transplant program. Transpl Int. 2005;18:1218-20.

181. Hagen EA, Lautenbach E, Olthoff K, et al. Low prevalence of colonization with vancomycin-resistant Enterococcus in patients awaiting liver transplantation. Am J Transplant. 2003;3:902-5.

182. Bakir M, Bova JL, Newell KA, et al. Epidemiology and clinical consequences of vancomycin-resistant enterococci in liver transplant patients. Transplantation. 2001;72:1032-7.

183. McNeil SA, Malani PN, Chenoweth CE, et al. Vancomycinresistant enterococcal colonization and infection in liver transplant candidates and recipients: a prospective surveillance study. Clin Infect Dis. 2006;42:195-203.

184. Orloff SL, Busch AM, Olyaei AJ, et al. Vancomycinresistant Enterococcus in liver transplant patients. Am J Surg. 1999; 177:418-22.

185. El-Khoury J, Fishman JA. Linezolid in the treatment of vancomycin-resistant enterococcus faecium in solid organ transplant recipients: report of a multicenter compassionate-use trial. Transpl Infect Dis. 2003;5:121-5.

186. Calfee DP, Salgado CD, Milstone AM, et al. Strategies to prevent methicillin-resistant Staphylococcus aureus transmission and infection in acute care hospitals: 2014 update. Infect Control Hosp Epidemiol. 2014;35(suppl 2):S108-32. 
187. Singh N, Paterson DL, Chang FY, Gayowski T, Squier C, Wagener MM, Marino IR. Methicillin-resistant Staphylococcus aureus: the other emerging resistant gram-positive coccus among liver transplant recipients. Clin Infect Dis. 2000;30:322-7.

188. Hand J, Patel G. Multidrug-resistant organisms in liver transplant: mitigating risk and managing infections. Liver Transpl. 2016;22(8):1143-53.

189. Frenette C, Sperlea D, Leharova Y, et al. Impact of an infection control and antimicrobial stewardship program on solid organ transplantation and hepatobiliary surgical site infections. Infect Control Hosp Epidemiol. 2016;37(12):1468-74.

190. Ramos A, Ortiz J, Asensio Á, et al. Risk factors for Clostridium difficile diarrhea in patients with solid organ transplantation. Prog Transplant. 2016;26(3):231-7.

191. Albright JB, Bonatti H, Mendez J, et al. Early and late onset Clostridium difficile-associated colitis following liver transplantation. Transpl Int. 2007;20(10):856-66.

192. Sullivan T, Weinberg A, Rana M, et al. The epidemiology and clinical features of Clostridium difficile infection in liver transplant recipients. Transplantation. 2016;100(9):1939-43.

193. Andes DR, Safdar N, Baddley JW, et al. The epidemiology and outcomes of invasive Candida infections among organ transplant recipients in the United States: results of the transplant-associated infection surveillance network (TRANSNET). Transpl Infect Dis 2016;18(6):921-31.

194. Silveira FP, Kusne S, the AST Infectious Diseases Community of Practice. Candida infections in solid organ transplantation. Am J Transplant. 2013;13(Suppl 4):220-7.

195. Husain S, Tollemar J, Dominguez EA, et al. Changes in the spectrum and risk factors for invasive candidiasis in liver transplant recipients: prospective, multicenter, case-controlled study. Transplantation. 2003;75(12):2023-9.

196. Singh N, Winston DJ, Limaye AP, et al. Performance characteristics of galactomannan and b-d-Glucan in high-risk liver transplant recipients. Transplantation. 2015;99(12):2543-50.

197. Pappas PJ, Kauffman CA, Andes DR. Clinical practice guideline for the management of candidiasis: 2016 update by the Infectious Diseases Society of America. Clin Infect Dis. 2016;62(4): e1-50.

198. Winston DJ, Pakrasi A, Busuttil RW. Prophylactic fluconazole in liver transplant recipients. A randomized, double-blind, placebocontrolled trial. Ann Intern Med. 1999;131(10):729-3.

199. Kusne S, Tobin D, Pasculle AW, et al. Candida carriage in the alimentary tract of liver transplant candidates. Transplantation. 1994;57(3):398-402.

200. Giannella M, Bartoletti M, Morelli M, et al. Antifungal prophylaxis in liver transplant recipients: one size does not fit all. Transpl Infect Dis. 2016;18(4):538-44.

201. Wajszczuk CP, Dummer JS, Ho M, et al. Fungal infections in liver transplant recipients. Transplantation. 1985;40(4):347-53.

202. Sganga G, Bianco G, Frongillo F, et al. Fungal infections after liver transplantation: incidence and outcome. Transplant Proc. 2014;46(7):2314-8.

203. Morgan J, Wannemuehler KA, Marr KA, et al. Incidence of invasive aspergillosis following hematopoietic stem cell and solid organ transplantation: interim results of a prospective multicenter surveillance program. Med Mycol. 2005;43(Suppl 1):S49-58.

204. Pappas PA, Alexander BD, Andes AR, et al. Invasive fungal infections among organ transplant recipients: results of the TransplantAssociated Infection Surveillance Network (TRANSNET). Clin Infect Dis. 2010;50(8):1101-11.

205. Fortún J, Martín-Dávila P, Moreno S, et al. Risk factors for invasive aspergillosis in liver transplant recipients. Liver Transpl. 2002;8(11):1065-70.

206. Paterson DL, Singh N. Invasive aspergillosis in transplant recipients. Medicine (Baltimore). 1999;78(2):123-38.
207. Singh N, Arnow P, Bonham A, et al. Invasive aspergillosis in liver transplant recipients in the 1990s. Transplantation. 1997;64(5):716-20.

208. Gavalda J, Len O, San Juan R, et al. Risk factors for invasive aspergillosis in solid-organ transplant recipients: a case-control study. Clin Infect Dis. 2005;41:52-9.

209. Singh N, Avery RK, Munoz P, et al. Trends in risk profiles for and mortality associated with invasive aspergillosis among liver transplant recipients. Clin Infect Dis. 2003;36:46-52.

210. Singh N, Husain S, the AST Infectious Diseases Community of Practice. Aspergillosis in solid organ transplantation. Am J Transplant. 2013;13(Suppl 4):228-41.

211. Husain S, Paterson DL, Studer SM, et al. Aspergillus galactomannan antigen in the bronchoalveolar lavage fluid for the diagnosis of invasive aspergillosis in lung transplant recipients. Transplantation. 2007;83(10):1330-6.

212. Patterson TF, Thompson GR, Denning DW, et al. Practice guidelines for the diagnosis and management of Aspergillosis: 2016 update by the Infectious Diseases Society of America. Clin Infect Dis. 2016;63(4):433-42.

213. Balogh J, Gordon Burroughs S, Boktour M, et al. Efficacy and cost-effectiveness of voriconazole prophylaxis for prevention of invasive aspergillosis in high-risk liver transplant recipients. Liver Transpl. 2016;22(2):163-70.

214. Winston DJ, Limaye AP, Pelletier S, et al. Randomized, doubleblind trial of anidulafungin versus fluconazole for prophylaxis of invasive fungal infections in high-risk liver transplant recipients. Am J Transplant. 2014;14(12):2758-64.

215. Perrella A, Esposito C, Amato G, et al. Antifungal prophylaxis with liposomal amphotericin B and caspofungin in high-risk patients after liver transplantation: impact on fungal infections and immune system. Infect Dis (Lond). 2016;48(2):161-6.

216. Fortún J, Muriel A, Martín-Dávila P, et al. Caspofungin versus fluconazole as prophylaxis of invasive fungal infection in high-risk liver transplantation recipients: a propensity score analysis. Liver Transpl. 2016;22(4):427-35.

217. Echenique IA, Ison MG. Update on donor-derived infections in liver transplantation. Liver Transpl. 2013;19(6):575-85.

218. Collins GM, Dubernard JM, Land W, et al. Procurement, preservation and allocation of vascularized organs. London: Kluwer Academic Publishers; 1997.

219. Doucette KE, Al-Saif M, Kneteman N, et al. Donor-derived bacteremia in liver transplant recipients despite antibiotic prophylaxis. Am J Transplant. 2013;13(4):1080-3.

220. Miceli MH, Gonulalan M, Perri MB, et al. Transmission of infection to liver transplant recipients from donors with infective endocarditis: lessons learned. Transpl Infect Dis. 2015;17(1):140-6.

221. Caballero F, Lopez-Navidad A, Domingo P, et al. Successful transplantation of organs retrieved from a donor with enterococcal endocarditis. Transpl Int. 1998;11:387-9.

222. Ison MG, Nalesnik MA. An update on donor-derived disease transmission in organ transplantation. Am J Transplant. 2011;11:1123-30.

223. Ison MG, Grossi P, AST Infectious Diseases Community of Practice. Donor-derived infections in solid organ transplantation. Am J Transplant. 2013;13:22-30.

224. Ison MG, Hager J, Blumberg E, et al. Donor-derived disease transmission events in the United States: data reviewed by the OPTN/UNOS Disease Transmission Advisory Committee. Am J Transplant. 2009;9(8):1929-35.

225. Singh N, Huprikar S, Burdette SD, for American Society of Transplantation, Infectious Diseases Community of Practice, Donor-Derived Fungal Infection Working Group, et al. Donorderived fungal infections in organ transplant recipients: guidelines of the American Society of Transplantation, Infectious Diseases Community of Practice. Am J Transplant. 2012;12:2414-28. 
226. Morris MI, Daly JS, Blumberg E, et al. Diagnosis and management of tuberculosis in transplant donors: a donor-derived infections consensus conference report. Am J Transplant. 2012;12:2288-300.

227. Wakelin SJ, Casey J, Robertson A, et al. The incidence and importance of bacterial contaminants of cadaveric renal perfusion fluid. Transpl Int. 2005;17:680-6.

228. Ruiz P, Gastaca M, Gonzalez J, et al. Incidence and clinical relevance of bacterial contamination in preservation solution for liver transplantation. Transplant Proc. 2009;41:2169-71.

229. Janny S, Bert F, Dondero F, et al. Microbiological findings of culture-positive preservation fluid in liver transplantation. Transpl Infect Dis. 2011;13:9-14.

230. Baddley JW, Schain DC, Gupte AA, et al. Transmission of Cryptococcus neoformans by organ transplantation. Clin Infect Dis. 2011;52:e94-8.

231. Kanj SS, Welty-Wolf K, Madden J, et al. Fungal infections in lung and heart-lung transplant recipients. Report of 9 cases and review of the literature. Medicine (Baltimore). 1996;75:142-56.

232. Ooi BS, Chen BT, Lim CH, et al. Survival of a patient transplanted with a kidney infected with Cryptococcus neoformans. Transplantation. 1971;11:428-9.

233. Centers for Disease Control and Prevention (CDC). Transplantation-transmitted tuberculosis - Oklahoma and Texas, 2007. MMWR Morb Mortal Wkly Rep. 2008;57:333-6.

234. Kotton CN, Lattes R, for AST Infectious Diseases Community of Practice. Parasitic infections in solid organ transplant recipients. Am J Transplant. 2009;9(suppl 4):S234-51.

235. Hamilton KW, Abt PL, Rosenbach MA, et al. Donor-derived Strongyloides stercoralis infections in renal transplant recipients. Transplantation. 2011;91:1019-24.

236. Chin-Hong PV, Schwartz BS, Bern C, et al. Screening and treatment of Chagas disease in organ transplant recipients in the United States: recommendations from the Chagas in Transplant Working Group. Am J Transplant. 2011;11:672-80.

237. Altclas JD, Barcan L, Nagel C, et al. Organ transplantation and Chagas disease. JAMA. 2008;299:1134.

238. Ljungman P, Griffiths P, Paya C. Definitions of cytomegalovirus infection and disease in transplant recipients. Clin Infect Dis. 2002;34:1094-7.

239. Paya CV, Hermans PE, Wiesner RH, et al. Cytomegalovirus hepatitis in liver transplantation: prospective analysis of 93 consecutive orthotopic liver transplantations. J Infect Dis. 1989;160:752-8.

240. Gane E, Saliba F, Valdecasas GJ, et al. Randomised trial of efficacy and safety of oral ganciclovir in the prevention of cytomegalovirus disease in liver-transplant recipients. The Oral Ganciclovir International Transplantation Study Group [corrected]. Lancet. 1997;350:1729-33.

241. Paya C, Humar A, Dominguez E, et al. Efficacy and safety of valganciclovir vs. oral ganciclovir for prevention of cytomegalovirus disease in solid organ transplant recipients. Am J Transplant. 2004;4:611-20.

242. Razonable RR, van Cruijsen H, Brown RA, et al. Dynamics of cytomegalovirus replication during preemptive therapy with oral ganciclovir. J Infect Dis. 2003;187:1801-8.

243. Singh N, Wannstedt C, Keyes L, et al. Who among cytomegalovirusseropositive liver transplant recipients is at risk for cytomegalovirus infection? Liver Transpl. 2005;11:700-4.

244. Arthurs SK, Eid AJ, Pedersen RA, et al. Delayed-onset primary cytomegalovirus disease after liver transplantation. Liver Transpl. 2007;13:1703-9.

245. Razonable RR. Epidemiology of cytomegalovirus disease in solid organ and hematopoietic stem cell transplant recipients. Am J Health Syst Pharm. 2005;62:S7-S13.

246. Razonable RR, Rivero A, Rodriguez A, et al. Allograft rejection predicts the occurrence of late-onset cytomegalovirus
(CMV) disease among CMV-mismatched solid organ transplant patients receiving prophylaxis with oral ganciclovir. J Infect Dis. 2001;184:1461-4.

247. Singh N. Late-onset cytomegalovirus disease as a significant complication in solid organ transplant recipients receiving antiviral prophylaxis: a call to heed the mounting evidence. Clin Infect Dis. 2005;40(5):704-8.

248. Kim JM, Kim SJ, Joh JW, et al. Early and delayed onset cytomegalovirus infection of liver transplant recipients in endemic areas. Transplant Proc. 2010;42:884-9.

249. Razonable RR, Paya CV. Infections and allograft rejection - intertwined complications of organ transplantation. Swiss Med Wkly. 2005;135:571-3.

250. O'Grady JG, Alexander GJ, Sutherland S, et al. Cytomegalovirus infection and donor/recipient HLA antigens: interdependent cofactors in pathogenesis of vanishing bile-duct syndrome after liver transplantation. Lancet. 1988;2:302-5.

251. Noack KB, Wiesner RH, Batts K, et al. Severe ductopenic rejection with features of vanishing bile duct syndrome: clinical, biochemical, and histologic evidence for spontaneous resolution. Transplant Proc. 1991;23:1448-51.

252. Ludwig J, Wiesner RH, Batts KP, et al. The acute vanishing bile duct syndrome (acute irreversible rejection) after orthotopic liver transplantation. Hepatology. 1987;7:476-83.

253. Gao LH, Zheng SS. Cytomegalovirus and chronic allograft rejection in liver transplantation. World $\mathrm{J}$ Gastroenterol. 2004;10(13):1857-61.

254. Pastacaldi S, Teixeira R, Montalto P, et al. Hepatic artery thrombosis after orthotopic liver transplantation: a review of nonsurgical causes. Liver Transpl. 2001;7:75-81.

255. Madalosso C, de Souza NF Jr, Ilstrup DM, et al. Cytomegalovirus and its association with hepatic artery thrombosis after liver transplantation. Transplantation. 1998;66:294-7.

256. Munoz-Price LS, Slifkin M, Ruthazer R, et al. The clinical impact of ganciclovir prophylaxis on the occurrence of bacteremia in orthotopic liver transplant recipients. Clin Infect Dis. 2004;39(9):1293-9.

257. George MJ, Snydman DR, Werner BG, et al. The independent role of cytomegalovirus as a risk factor for invasive fungal disease in orthotopic liver transplant recipients. Boston center for liver transplantation cmvig-study group. Cytogam, Medimmune, Inc. Gaithersburg, Maryland. Am J Med. 1997;103(2): 106-13.

258. Walker RC, Marshall WF, Strickler JG, et al. Pretransplantation assessment of the risk of lymphoproliferative disorder. Clin Infect Dis. 1995;20(5):1346-53.

259. Stratta RJ, Shaefer MS, Markin RS, et al. Clinical patterns of cytomegalovirus disease after liver transplantation. Arch Surg. 1989;124(12):1443-50.

260. Razonable RR. Cytomegalovirus infection after liver transplantation: current concepts and challenges. World J Gastroenterol. 2008;14(31):4849-60. Epub 2008/08/30.

261. Singh N, Dummer JS, Kusne S, et al. Infections with cytomegalovirus and other herpesviruses in 121 liver transplant recipients: transmission by donated organ and the effect of OKT3 antibodies. J Infect Dis. 1988;158(1):124-31.

262. Razonable RR, Humar A, AST Infectious Diseases Community of Practice. Cytomegalovirus in solid organ transplantation. Am J Transplant. 2013;13(Suppl 4):93-106.

263. Mumtaz K, Faisal N, Husain S, et al. Universal prophylaxis or preemptive strategy for cytomegalovirus disease after liver transplantation: a systematic review and meta-analysis. Am J Transplant. 2015;15(2):472-81.

264. Marcelin JA, Beam E, Razonable RR. Cytomegalovirus infection in liver transplant recipients: updates on clinical management. World J Gastroenterol. 2014;20(31):10658-67. 
265. Kostakis ID, Sotiropoulos GC, Kouraklis G. Pneumocystis jirovecii pneumonia in liver transplant recipients: a systematic review. Transplant Proc. 2014;46(9):3206-8.

266. Panel on Opportunistic Infections in HIV-Infected Adults and Adolescents. Guidelines for the prevention and treatment of opportunistic infections in HIV-infected adults and adolescents: recommendations from the Centers for Disease Control and Prevention, the National Institutes of Health, and the HIV Medicine Association of the Infectious Diseases Society of America. Available at http://aidsinfo.nih.gov/contentfiles/lvguidelines/adult_oi.pdf. Accessed 2/2018.

267. Martina SI, Fishman JA, the AST Infectious Diseases Community of Practice. Pneumocystis pneumonia in solid organ transplantation. Am J Transplant. 2013;13(Suppl 4):272-9.

268. Baddley JW, Forrest GN, the AST Infectious Diseases Community of Practice (2013). Cryptococcosis in solid organ transplantation. Am J Transplant. 2013;13(Suppl 4):242-9.

269. Singh N, Dromer F, Perfect JR, et al. Cryptococcosis in solid organ transplant recipients: current state of the science. Clin Infect Dis. 2008;47:1321-7.

270. Husain S, Wagener MM, Singh N. Cryptococcus neoformans infection in organ transplant recipients: variables influencing clinical characteristics and outcome. Emerg Infect Dis. 2001;7: 375-81.

271. Singh N, Alexander BD, Lortholary O, et al. Cryptococcus neoformans in organ transplant recipients: impact of calcineurininhibitor agents on mortality. J Infect Dis. 2007;195:756-64.

272. Vilchez RA, Fung J, Kusne S. Cryptococcosis in organ transplant recipients: an overview. Am J Transplant. 2002;2:575-80.

273. Jabbour N, Reyes J, Kusne S, et al. Cryptococcal meningitis after liver transplantation. Transplantation. 1996;61:146-9.

274. Perfect JR, Dismukes WE, Dromer F, et al. Clinical practice guidelines for the management of cryptococcal disease: 2010 update by the Infectious Diseases Society of America. Clin Infect Dis. 2010;50:291-322.

275. Freifeld A, Iwen PC, Lesiak BL, et al. Histoplasmosis in solid organ transplant recipients at a large Midwestern university transplant center. Transpl Infect Dis. 2005;7:109-15.

276. Grim S, Proia L, Miller R, et al. A multicenter study of histoplasmosis and blastomycosis after solid organ transplantation. Transpl Infect Dis. 2012;14:17-23.

277. Batista M, Pierrotti LC, Abdala E, et al. Endemic and opportunistic infections in Brazilian solid organ transplant recipients. Tropical Med Int Health. 2011;16:1134-42.

278. Cuellar-Rodriguez J, Avery RK, Lard M, et al. Histoplasmosis in solid organ transplant recipients: 10 years of experience at a large transplant center in an endemic area. Clin Infect Dis. 2009;49:710-6.

279. Miller R, Assi M, AST Infectious Diseases Community of Practice. Endemic fungal infections in solid organ transplantation. Am J Transplant. 2013;13:250-61.

280. Vucicevic D, Carey EJ, Blair JE. Coccidioidomycosis in liver transplant recipients in an endemic area. Am J Transplant. 2011;11:111-9.

281. Blair J. Coccidioidomycosis in patients who have undergone transplantation. Ann N Y Acad Sci. 2007;1111:365-76.

282. Holt C, Winston DJ, Kubak B, et al. Coccidioidomycosis in liver transplant patients. Clin Infect Dis. 1997;24:216-21.

283. Blair J, Logan JL. Coccidioidomycosis in solid organ transplantation. Clin Infect Dis. 2001;33:1536-44.

284. Gauthier GM, Safdar N, Klein BS, Andes DR. Blastomycosis in solid organ transplant recipients. Transpl Infect Dis. 2007;9:310-7.

285. Pedersen M, Seetharam A. Infections after orthotopic liver transplantation. J Clin Exp Hepatol. 2014;4(4):347-60.

286. Hamandi B, Husain S, Grootendorst P, et al. Clinical and microbiological epidemiology of early and late infectious complications among solid-organ transplant recipients requiring hospitalization. Transpl Int. 2016;29(9):1029-38.

287. Kumar D, Prasad GV, Zaltzman J, et al. Community-acquired West Nile virus infection in solid-organ transplant recipients. Transplantation. 2004;77(3):399.

288. Clark NM, Reid GE. ATS infectious, disease community, of practice Nocardia infections in solid organ transplantation. Am J Transplant. 2013;13:83-92.

289. Husain S, McCurry K, Dauber J, et al. Nocardia infection in lung transplant recipients. J Heart Lung Transplant. 2002;21: 354-9.

290. Peleg AY, Husain S, Qureshi ZA, et al. Risk factors, clinical characteristics, and outcome of Nocardia infection in organ transplant recipients: a matched case-control study. Clin Infect Dis. 2007:44:1307-14.

291. Coussement J, Lebeaux D, van Delden C, et al. Nocardia infection in solid organ transplant recipients: a multicenter European casecontrol study. Clin Infect Dis. 2016;63:338-45.

292. Park BJ, Pappas PG, Wannemuehler KA, et al. Invasive non-aspergillus mold infections in transplant recipients, United States, 2001-2006. Emerg Infect Dis. 2011;17(10): 1855-64.

293. Silveira FP, Husain S. Fungal infections in solid organ transplantation. Med Mycol. 2007;45:305-20.

294. Singh N, Chang FY, Gayowski T, et al. Infections due to dematiaceous fungi in organ transplant recipients: case report and review. Clin Infect Dis. 1997;24:369-74.

295. Eshraghian A, Imanieh MH, Dehghani SM, et al. Post-transplant lymphoproliferative disorder after liver transplantation: incidence, long-term survival and impact of serum tacrolimus level. World J Gastroenterol. 2017;23:1224-32.

296. Penn I. Posttransplantation de novo tumors in liver allograft recipients. Liver Transpl Surg. 1996;2:52-9.

297. Green M, Michaels MG. Epstein-Barr virus infection and posttransplant lymphoproliferative disorder. Am J Transplant. 2013;13(Suppl 3):41-54.

298. Singh N, Paterson DL. Mycobacterium tuberculosis infection in solid-organ transplant recipients: impact and implications for management. Clin Infect Dis. 1998;27(5):1266-77.

299. Nahid P, Dorman SE, Alipanah N, et al. Official American Thoracic Society/Centers for Disease Control and Prevention/ Infectious Diseases Society of America Clinical Practice Guidelines: treatment of drug-susceptible tuberculosis. Clin Infect Dis. 2016;63(7):e147-95.

300. Weltermann B, Herwig A, Dehnen D, et al. Vaccination status of pneumococcal and other vaccines in 444 liver transplant patients compared to a representative population sample. Ann Transplant. 2016;21:200-7.

301. Roni DA, Pathapati RM, Kumar AS, et al. Safety and efficacy of hepatitis B vaccination in cirrhosis of liver. Adv Virol. 2013;2013:196704. Epub 2013 Jun 6.

302. Danziger-Isakov L, Kumar D, the AST Infectious Diseases Community of Practice. Vaccination in solid organ transplantation. Am J Transplant. 2013;13(Suppl 4):311-7.

303. Bratzler DW, Dellinger EP, Olsen KM, et al. Clinical practice guidelines for antimicrobial prophylaxis in surgery. Am J HealthSyst Pharm. 2013;70:195-283.

304. Todo S, Demetris AJ, Van Thiel D, et al. Orthotopic liver transplantation for patients with hepatitis B virus-related liver disease. Hepatology. 1991;13(4):619-26.

305. Lau JY, Bain VG, Davies SE, et al. High-level expression of hepatitis B viral antigens in fibrosing cholestatic hepatitis. Gastroenterology. 1992;102(3):956-62.

306. Marzano A, Gaia S, Ghisetti V, et al. Viral load at the time of liver transplantation and risk of hepatitis B virus recurrence. Liver Transpl. 2005;11(4):402-9. 
307. Abdelmalek MF, Pasha TM, Zein NN, et al. Subclinical reactivation of hepatitis B virus in liver transplant recipients with past exposure. Liver Transpl. 2003;9(12):1253-7.

308. Wright TL, Donegan E, Hsu HH, et al. Recurrent and acquired hepatitis $\mathrm{C}$ viral infection in liver transplant recipients. Gastroenterology. 1992;103(1):317-22.

309. Lake JR, Shorr JS, Steffen BJ, et al. Differential effects of donor age in liver transplant recipients infected with hepatitis B, hepatitis C and without viral hepatitis. Am J Transplant. 2005;5(3):549-57.

310. Freeman RB, Tran S, Lee YM, et al. Serum hepatitis C RNA titers after liver transplantation are not correlated with immunosuppression or hepatitis. Transplantation. 1996;61(4):542-6.

311. Berenguer M. Natural history of recurrent hepatitis C. Liver Transpl. 2002;8(10 Suppl 1):S14-8.

312. Verna EC, Abdelmessih R, Samomao MA, et al. Cholestatic hepatitis $\mathrm{C}$ following liver transplantation: an outcome-based histological definition, clinical predictors, and prognosis. Liver Transpl. 2013;19(1):78-88.

313. McGory RW, Ishitani MB, Oliveira WM, et al. Improved outcome of orthotopic liver transplantation for chronic hepatitis B cirrhosis with aggressive passive immunization. Transplantation. 1996;61(9):1358-64.

314. Charlton M, Everson GT, Flamm SL, et al. Ledipasvir and sofosbuvir plus ribavirin for treatment of $\mathrm{HCV}$ infection in patients with advanced liver disease. Gastroenterology. 2015;149(3):649-59.

315. O'Leary JG, Brown K, Burton J, et al. Efficacy and safety of simeprevir and sofosbuvir with and without ribavirin for 12 weeks in subjects with recurrent genotype 1 hepatitis C postorthotopic liver transplant: the GALAXY study. Transpl Int. 2017;30(2):196-208.

316. Brown RS, O'Leary J, Redy KR, et al. Interferon-free therapy for genotype 1 hepatitis $\mathrm{C}$ in liver transplant recipients: real world experience from the hepatitis $\mathrm{C}$ therapeutic registry and research network. Liver Transpl. 2016;22(1):24-33.

317. Fontana RJ, Brown RS, Moreno-Zamora A, et al. Daclatasvir combined with sofosbuvir or simeprevir in liver transplant recipients with severe recurrent hepatitis $\mathrm{C}$ infection. Liver Transpl. 2016;22:446-58.

318. Kwo PY, Mantry PS, Coakley E, et al. An interferon-free antiviral regimen for $\mathrm{HCV}$ after liver transplantation. N Engl J Med. 2014;371(25):2375-82.

319. Kemmer NM, Miskovsky EP. Hepatitis A. Infect Dis Clin N Am. 2000;14(3):605-15.

320. Arslan M, Wiesner RH, Poterucha JJ, et al. Hepatitis A antibodies in liver transplant recipients: evidence for loss of immunity posttransplantation. Liver Transpl. 2000;6(2):191-5.

321. Arslan M, Wiesner RH, Poterucha JJ, et al. Safety and efficacy of hepatitis A vaccination in liver transplantation recipients. Transplantation. 2001;72(2):272-6.

322. Askling HH, Rombo L, van Vollenhoven R, et al. Hepatitis A vaccine for immunosuppressed patients with rheumatoid arthritis: a prospective, open-label, multi-centre study. Travel Med Infect Dis. 2014;12(2):134-42.

323. Garcia Garrido HM, Wieten RW, Grobusch MP, et al. Response to hepatitis A vaccination in immunocompromised travelers. J Infect Dis. 2015;212(3):378-85
324. Patra S, Kumar A, Trivedi SS, et al. Maternal and fetal outcomes in pregnant women with acute hepatitis E virus infection. Ann Intern Med. 2007;147(1):28-33.

325. Hamid SS, Atiq F, Shehzad A, et al. Hepatitis E virus superinfection in patients with chronic liver disease. Hepatology. 2002;36(2):474-8.

326. Ramachandran J, Eapen C, Kang G, et al. Hepatitis E superinfection produces severe decompensation in patients with chronic liver disease. J Gastroenterol Hepatol. 2004;19(2): 134-8.

327. Coilly A, Haim-Boukobza B, Roche TM, Antonini A, et al. Posttransplantation hepatitis E: transfusion-transmitted hepatitis rising from the ashes. Transplantation. 2013;96(2):e4-6.

328. Schlosser B, Stein A, Neuhaus R, et al. Liver transplant from a donor with occult HEV infection induced chronic hepatitis and cirrhosis in the recipient. J Hepatol. 2012;56(2):500-2.

329. Haagsma EB, van den Berg AP, Porte RJ, et al. Chronic hepatitis $\mathrm{E}$ virus infection in liver transplant recipients. Liver Transpl. 2008;14(4):547-53.

330. Kamar N, Garrouste C, Haagsma EB, et al. Factors associated with chronic hepatitis in patients with hepatitis $\mathrm{E}$ virus infection who have received solid organ transplants. Gastroenterology. 2011;140(5):1481-9.

331. Pischke S, Suneetha PV, Baechlein C, et al. Hepatitis E virus infection as a cause of graft hepatitis in liver transplant recipients. Liver Transpl. 2010;16(1):74-82.

332. Pischke S, Behrendt P, Manns MP, et al. HEV-associated cryoglobulinaemia and extrahepatic manifestations of hepatitis E. Lancet Infect Dis. 2014;14(8):678-9.

333. Legrand-Abravanel F, Kamar N, Sandres-Saune K, et al. Hepatitis E virus infection without reactivation in solid-organ transplant recipients, France. Emerg Infect Dis. 2011;17(1):30-7.

334. Buffaz C, Scholtes C, Dron AG, et al. Hepatitis E in liver transplant recipients in the Rhone-Alps region in France. Eur J Clin Microbiol Infect Dis. 2014;33(6):1037-43.

335. Aggarwal R. Hepatitis E: clinical presentation in disease-endemic areas and diagnosis. Semin Liver Dis. 2013;33(1):30-40.

336. Wang Y, Zhou X, Debing Y, et al. Calcineurin inhibitors stimulate and mycophenolic acid inhibits replication of hepatitis $\mathrm{E}$ virus. Gastroenterology. 2014;146(7):1775-83.

337. Zhou X, Wang Y, Metselaar HJ, et al. Rapamycin and everolimus facilitate hepatitis E virus replication: revealing a basal defense mechanism of PI3K-PKB-mTOR pathway. J Hepatol. 2014;61(4):746-54.

338. Pischke S, Stiefel P, Franz B, et al. Chronic hepatitis E in heart transplant recipients. Am J Transplant. 2012;12(11): 3128-33.

339. Kamar N, Izopet J, Tripon S, et al. Ribavirin for chronic hepatitis $\mathrm{E}$ virus infection in transplant recipients. $\mathrm{N}$ Engl $\mathrm{J}$ Med. 2014;370(12):1111-20.

340. Kamar N, Rostaing L, Abravanel F, et al. Ribavirin therapy inhibits viral replication on patients with chronic hepatitis $\mathrm{E}$ virus infection. Gastroenterology. 2010;139(5):1612-8.

341. Mallet V, Nicand E, Sultanik P, et al. Brief communication: case reports of ribavirin treatment for chronic hepatitis E. Ann Intern Med. 2010;153(2):85-9. 